\title{
COMPUTER SIMULATION OF RECRYSTALLIZATION-III. INFLUENCE OF A DISPERSION OF FINE PARTICLES
}

\section{A. D. ROLLETT ${ }^{1}$, D. J. SROLOVITZ ${ }^{2}$, M. P. ANDERSON ${ }^{3}$ and R. D. DOHERTY}

'Materials Science and Technology Division, Los Alamos National Laboratory, Los Alamos, NM 87545, '2Department of Materials Science and Engineering, University of Michigan, Ann Arbor MI 48109, ${ }^{3}$ Exxon Research and Engineering Company, Annandale, NJ 08801 and ${ }^{4}$ Department of Materials Science and Engineering, Drexel University, Philadelphia, PA 19104, U.S.A.

\section{(Received 2 October 1990; in revised form 2 March 1992)}

\begin{abstract}
Two-dimensional Monte Carlo simulations of recrystallization have been carried out in the presence of incoherent and immobile particles for a range of different particle fractions, a range of stored energies and a range of densities of potential nuclei (embryos). For stored energies greater than a critical value $(H / J>1)$ the recrystallization front can readily pass the particles leading to a random density of particles on the front and a negligible influence of particles on the recrystallization kinetics. At lower stored energies the particles pin the recrystallization front leading to incomplete recrystallization. However at very low particle fractions, when the new grain has grown much larger than the matrix grains, before meeting any particles, the new grains can complete the consumption of the deformed grains giving complete "recrystallization" by a process that appears to be similar to abnormal grain growth. Particles are, as reported previously, very effective at pinning grain boundaries, both of the deformed and recrystallized grains, when boundaries migrate under essentially the driving force of boundary energy alone. Such boundaries show a density of particles that rises rapidly from the random value found at the start of the simulation. As a consequence, particles very strongly inhibit normal grain growth after recrystallization. Such growth can only occur if the as-recrystallized grain size is less than the limiting grain size seen in the absence of recrystallization. Under these circumstances a small increment of grain growth occurs until the grain boundaries once again acquire a higher than random density of particles.
\end{abstract}

Résumé-On effectue des simulations de Monte-Carlo à deux dimensions pour étudier la recristallisation en présence de particules incohérentes et immobiles pour une gamme de fractions différentes de particules, une gamme d'énergies stockées imposées et une gamme de densité de germes potentiels (embryons). Pour des énergies stockées supérieures à une valeur critique $(H / J>1)$, le front de recristallisation peut facilement traverser les particules, conduisant à une densité aléatoire de particules sur le front et à une influence négligeable des particules sur la cinétique de recristallisation. Pour de plus basses énergies stockées les particules épinglent le front de recristallisation, conduisant à une recristallisation incomplète. Cependant, pour de très faibles fractions de particules, lorsque le nouveau grain est devenu beaucoup plus grand que ceux de la matrice, avant de recontrer une particule quelconque, les nouveaux grains peuvent achever l'absorption des grains déformes, créant une "recristallisation" complète par un processus qui est semblable à celui de la croissance anormale des grains. Comme on l'a déjà signalé, les particules sont très efficaces pour l'épinglage des joints de grains, tant déformés que recristallisés, lorsque les joints migrent principalement sous l'effet de la force motrice de l'énergie intergranulaire seule. De tels joints révèlent une densité de particules qui s’ëlève rapidement depuis la valeur aléatoire trouvée au début de la simulation. En conséquence, les particules empêchent très fortement une croissance normale des grains après la recristallisation. Une telle croissance ne peut se produire que si la taille des grains recristallisés est inférieure à la taille limite du grain en l'absence de recristallisation. Dans ce cas, une petite augmentation de la croissance des grains se produit, jusqu'à ce que les joints de grains acquièrent une densité de particules supérieure à la densité aléatoire.

Zusammenfassung-Die Rekristallisation in Gegenwart von inkohärenten und unbeweglichen Teilchen wird für verschiedene Teilchenanzahldichten. gespeicherte Energien und Dichten möglicher Keime (Embryos) mittels einer zweidimensionalen Monte-Carlo-Rechnung simuliert. Bei einer gespeicherten Energie oberhalb einem kritischen Wert $(H / J>1)$ kann die Rekristallisationsfront die Teilchen einfach passieren, welches zu einer zufälligen Dichte von Teilchen auf der Front und zu einem vernachlässigbaren Einfluß der Teilchen auf die Rekirstallisationskinetik führt. Bei geringerer gespeicherter Energie verankern die Teilchen die Rekristallisationsfront, welches zu unvollständiger Rekristallisation führt. Allerdings können im Falle sehr kleiner Teilchendichten die neuen Körner vor dem Treffen auf Teilchen viel größer werden als die Matrixkörner und die verformten Körner vollständig aufzehren: das führt zu einer vollständigen "Rekristallisation", die ähnlich dem abnormalen Kornwachstum ist. Teilchen können, wie früher ausgeführt, Korngrenzen sowohl der verformten wie auch der rekristallisierten Körner sehr wirksam verankern, wenn die Korngrenzen im wesentlichen unter der treibenden Kraft durch die Korngrenzenergie alleine wandern. Solche Korngrenzen weisen eine Teilchendichte auf, die rasch von dem zufäligen, zu Beginn der Simulation beobachteten Wert aus ansteigt. Daher behindern die Teilchen sehr stark das normale Kornwachstum nach der Rekristallisation. Ein solches Wachstum kann nur stattfinden, wenn die Größe der rekristallisierten Körner kleiner ist als die in Abwesenheit der Rekristallisation geltende Grenze der Korngröße. Unter diesen Umständen tritt eine kleine Zunahme des Kornwachstums auf, bis die Korngrenzen wiederum eine höhere Teilchendichte als die zufällige Dichte aufgenommen haben. 


\section{INTRODUCTION}

The effect of second phase particles on primary recrystallization is profound. This topic has been extensively investigated since the early 1960s [1] and has been the subject of numerous reviews [2-4]. An interesting feature of recrystallization in the presence of particles is that in some cases particles retard recrystallization, while in others the recrystallization kinetics are accelerated. The reasons for this variable effect are associated with the competition between retardation by particles of grain boundary migration and the particle stimulated nucleation of recrystallized grains. The key conclusions to be drawn from the reviews of the topic are as follows:

(1) Acceleration of recrystallization occurs when large $(>1 \mu \mathrm{m})$ particles are present. Humphreys [5] has shown that large particles are surrounded by a deformation zone in which there is a higher stored energy and larger misorientations than in the matrix. These factors lead to a significantly higher nucleation and growth rate near the particles. For a sufficiently high particle density, smaller recrystallized grain sizes can be achieved than in the corresponding single-phase material. This phenomenon is known as particle stimulated nucleation (PSN) and is of great importance in commercial materials. It depends, however, on heterogeneities in the deformed microstructure; the simulations described in this paper assume uniform, homogeneous stored energy, however, and so PSN will not be discussed further.

(2) Retardation of recrystallization occurs in the presence of fine particles where the mean inter-particle spacing $\left(\Delta_{3}\right)$ is of the order of the sub-grain size, typically $<1 \mu \mathrm{m}$. In this case the fine dispersion retards coarsening of the sub-grain structure which, in turn, inhibits the nucleation of recrystallized grains.

(3) The growth of recrystallized grains is relatively unaffected by the presence of particles on any scale, suggesting that retardation of recrystallization is largely a consequence of the inhibition of nucleation. This point was clearly demonstrated in the experiments of Doherty and Martin [1].

The present work builds on a series of successful simulations of recrystallization $[6-8]$ and grain growth [9-15], especially the effects of particles on limiting grain growth $[16,17]$. The results presented here directly address the issue of retardation of recrystallization by particle drag on the recrystallization front. In general they indicate a sharp transition from the inhibition of nucleation suggested by point
(2) above at low stored energies, to the unaffected growth of new grains suggested by point (3) at high stored energies. This study, therefore, requires a brief discussion of recent, non-classical results for the effects of pinning particles on grain boundary motion as illustrated by recent experiments and the earlier simulations [16].

The classical analysis of the effects of second phase particle on grain growth is due to Zener [18]. The Zener analysis, which is based on the assumption of a random correlation of particles with grain boundaries $[16,19]$, predicts that the particle dispersion results in a grain boundary drag pressure, $Z$

$$
Z=\frac{3 f \gamma}{2 r}
$$

where $f$ is the volume fraction of spherical particles of radius $r$ which are incoherent with respect to the matrix, and $\gamma$ is the grain boundary energy. For a reasonable choice of grain boundary energy, $0.5 \mathrm{~J} / \mathrm{m}^{2}$, a volume fraction of $1 \%$, and a particle size of $0.05 \mu \mathrm{m}$, the drag pressure is $0.15 \mathrm{MPa}$. The driving pressure for recrystallization [20] is approximated as

$$
P=0.5 \mu h^{2} \rho
$$

where $\rho$ is the dislocation density, $\mu$ is the shear modulus and $b$ is the Burgers vector of the dislocations. For a cold-rolled metal, $P$ is typically of the order of $10 \mathrm{MPa}$ and is clearly much larger than the particle drag pressure. This supports the conclusion that particles have little effect on the motion of boundaries between recrystallized and unrecrystallized regions. Experimental support for this is found in the hardness indent experiments of Ashby et al. [21]. In these experiments, annealed $\mathrm{Al}-3 \mathrm{wt} \% \mathrm{Cu}$ and $\mathrm{Cu}-2 \mathrm{wt} \% \mathrm{Co}$ alloys containing $\theta$ and $\mathrm{Co}$ particles, respectively, were indented to give a dislocation density that decreased with distance from the indent. The specimens were then annealed so that nucleation occurred at the indents and growth took place until the (constant) particle drag balanced the driving pressure, which decreased with distance from the indent. The magnitude of the particle drag, measured in this way, was found to be in agreement with the results of the Zener analysis. The number density of particles on the recrystallization front can be seen to be in agreement with the density predicted from a random correlation of boundaries with particles, as assumed in the Zener analysis.

In the last few years the validity of the Zener prediction, as applied to the inhibition of normal grain growth, has been challenged on theoretical grounds and on the basis of experimental studies, predominantly in the ceramic literature. For example, a recent review by Olgaard and Evans [22] showed both a wide scatter in the experimentally determined particle pinned grain size data and also a consistent trend such that pinned grain sizes were found to be much smaller than those predicted by the Zener analysis at low volume fractions. Computer 
simulations in both two [11] and three [17] dimensions have also indicated that particles are very much more effective at inhibiting normal grain growth than would be expected on the basis of the Zener analysis. This topic has recently been reviewed by Doherty et al. $[16,19]$ who pointed out that the simulations displayed a much higher correlation of particles with grain boundaries than predicted on the basis of the assumption used by Zener (i.e. that the number of particles intersected by a unit area of grain boundary is equal to the number of intersections between randomly distributed particles and a randomly located plane). Since the particle drag effect arises from the reduction in grain boundary energy when a particle intersects the grain boundary, it is not surprising that in the small driving pressure (energy) process of normal grain growth a higher than random correlation of particles with grain boundaries is found. It should be noted that a nearly equivalent viewpoint - that of the removal of the boundary curvature, required for grain growth, by particleshas been very successful in interpreting the enhanced inhibition of grain growth by particles [11, 15, 23].

The classical, Zener, prediction of the limiting grain size, $R_{\infty}$, in grain growth is

$$
R_{\infty}=\frac{2 r}{3 f} \text {. }
$$

The crucial results of the computer simulations, however, are that in two dimensions, the dependence of the limiting grain size on volume fraction is best fit by

$$
R_{\infty}=\frac{1.7 r}{\sqrt{f}}
$$

In three dimensions the volume fraction dependence is fit by

$$
R_{\infty}=\frac{2.8 r}{\sqrt[3]{f}}
$$

These results can be rationalized by considering the limiting grain size to be related to the mean nearest neighbor spacing of the particles, whose volume fraction dependence is proportional to $f^{-1 / 2}$ in two dimensions and $f^{-1 / 3}$ in three dimensions [24]. In (three-dimensional) microstructural terms, such a result can be obtained by assuming that the majority of particles lie either on grain edges (giving $f^{-1 / 2}$ ) or on grain corners (giving $f^{-1 / 3}$ ), see the review by Doherty et al. [16]. These results differ from the classical one and tend to explain the results quoted above.

The main consequence for retardation of recrystallization is that the pinning effects of a particle dispersion on nucleation are stronger than expected based upon the Zener approach. A short review of the nucleation of recrystallization follows to illustrate this point.

It is now generally accepted that classical homogeneous nucleation is impossible in recrystallization
[25]. Standard theory gives the size of the critical nucleus, $r_{\text {crit. }}$, as

$$
r_{\text {crit. }}=\frac{2 \gamma}{\Delta G}
$$

where $\Delta G$ is the difference in free energy (per unit volume) between the material in the recrystallization nucleus and that in the surrounding matrix, $\gamma$ is the high angle grain boundary energy (per unit area), and the free energy for nucleation, $\Delta F^{*}$ as

$$
\Delta G^{*}=\frac{16 \pi \gamma^{3}}{3 \Delta G^{2}}
$$

For a reasonable value of $\gamma=0.5 \mathrm{~J} \cdot \mathrm{m}^{-2}$, and a driving pressure of $10 \mathrm{MPa}$ as estimated above, the critical size for a recrystallization nucleus or embryonic new grain is about $0.1 \mu \mathrm{m}$. As a consequence, $\Delta G^{*} \gg k T$ which shows that nucleation requires a pre-existing feature of the deformed microstructure, such as a relatively large subgrain. Moreover, for the subgrain to grow it requires part of its boundary to be mobile which, in turn, implies that a large crystallographic misorientation ( $>20^{\circ}$ ) across the boundary must be present. In order to achieve nucleation, some of the subgrains must grow to the extent that they are larger than the critical size $r^{*}$. The role of small particles in inhibiting recrystallization appears to be in preventing the coarsening of the subgrain structure. Doherty and Martin [1] showed that a fine incoherent precipitate in an $\mathrm{Al}-\mathrm{Cu}$ alloy very effectively inhibits nucleation of recrystallization. In the $\mathrm{Al}-\mathrm{Al}_{2} \mathrm{O}_{3}$ system, where the second phase is also incoherent with respect to the matrix, Nobili and DeMaria [26] showed a similar retardation of recrystallization. They also showed that the recrystallization temperature increased with oxide content, i.e. that the retardation increased with increasing volume fraction of second phase. When the second phase particles are coherent with the matrix, the passage of a recrystallization front requires a large orientation change of the matrix which in turn means that the particle will no longer be coherent. This suggestion has been confirmed in a model system [21], nickelbased super alloys [27], aluminum alloy [28] and steels with carbides having the $\mathrm{NaCl}$ structure [29] (see review by Doherty [30]).

The retardation of recrystallization by fine particles is most likely the result of pinning of subgrain boundaries by the particles and the consequent prevention of nucleation. This view is supported by the experimental observation that when retardation occurs but recrystallization nevertheless goes to completion, the grain size is very coarse-indicating that very few nuclei appeared. Hutchinson and Duggan [31] appear to have been the first to recognize that the inhibition of recrystallization by closely spaced fine particles gives rise, in sub-grain growth, to exactly the same type of failure of the Zener analysis as that now seen in the computer simulations of normal grain growth. That is they pointed out that there was a very 
much higher than random correlation of particles with sub-grain boundaries in the recovered microstructure of deformed alloys.

Some reviewers (e.g. Humphreys [3] and Baker and Martin [32]) have used the Zener--Smith analysis to deduce that the particles do not produce sufficient drag to be the sole cause of retardation. The discussion of particle pinning above, however, indicates that this may not be the case. A possible alternative explanation for the role of fine dispersions was suggested by Humphreys [3] who experimentally demonstrated that the addition of dispersions of fine particles often produce more uniform, diffuse dislocation substructures and fewer large local misorientations. This lack of large misorientations can clearly contribute to the decrease in nucleation density. This suggestion will not be addressed in the present work.

The research reported in the present paper makes use of the Monte Carlo computer simulation model for microstructural evolution, previously applied to grain growth and recrystallization [6-15] in two dimensions, to study recrystallization of a polycrystalline material containing a dispersion of particles. A range of particle (area) fractions and different stored energies were examined. The different stored energies represent different dislocation densities remaining after deformation, as discussed above. Nucleation was imposed on the system at the start of the recrystallization (site saturated nucleation) by inserting different densities of embryos into the lattice. Imposing nucleation in this way meant that nucleation itself, and the influence of particles on nucleation, was not studied. That is to say, this study focused on the growth of new grains during recrystallization as a function of three parameters: stored energy, nucleation density and particle fraction. In addition, we examined the fraction of particles on grain boundaries throughout the course of recrystallization as the driving force changes from that associated with dislocations [equation (2)] to grain boundary curvature driven growth [see equation (1)]. The motivation for focusing on growth, as opposed to nucleation, in recrystallization arises from limitations of the Monte Carlo technique. Further discussion of this point is found at the end of the paper.

\section{SIMULATION PROCEDURE}

The simulation procedure employed in the present study is essentially identical to that employed in our previous simulations of recrystallization in homogeneous materials. In short, a continuum microstructure is mapped onto a two-dimensional triangular lattice containing $N=40,000$ sites. Each lattice site is assigned a number $S_{i}$ which corresponds to the orientation of the grain in which it is embedded. Lattice sites which are adjacent to sites having different grain orientations are regarded as being separated by a grain boundary, whilst a site surrounded by sites with the same orientation is in the grain interior.
Each unlike pair of nearest neighbors is assigned an energy $J$ so that the total energy of the system is calculated as

$$
E=\frac{J}{2} \sum_{i}^{N} \sum_{j}^{n n}\left(1-\delta_{S_{i} S_{j}}\right)
$$

where the sum on $i$ is over all $N$ sites in the system, the sum on $j$ is over the nearest neighbor sites $(n n=6)$ of site $i$, and $\delta_{i j}$ is the Kronecker delta. Grain growth is simulated by permitting the orientation of a particular site to change, provided that the total energy is decreased or remains unchanged as a result of the change in orientation. The sites in the system are sampled in a random order and $N$ such reorientation attempts are defined as one Monte Carlo step (MCS). The number of Monte Carlo steps is taken as being proportional to time. Particles are introduced into the simulation as sites which have an orientation different from any of the grains and which can not be reoriented during the course of the simulation. This assumption results in an equality of the particlematrix interfacial energy and the grain boundary energy, which is reasonable for particles that are incoherent with respect to the matrix. Also, the particles cannot move through the lattice which means that grain boundary drag of particles is not permitted [33].

Primary recrystallization in the presence of particles is modeled by incorporating an additional term into the energy expression equation (1) which accounts for the energy stored in the matrix, generally in the form of dislocations. This additional energy is present in the unrecrystallized grains but is absent in the recrystallized grains. The total energy $E$ is then calculated as

$$
E=\sum_{i}^{N}\left[H f\left(S_{i}\right)+\frac{J}{2} \sum_{j}^{n n}\left(1-\delta_{S_{i} S_{j}}\right)\right]
$$

where the function $f(S)$ is unity for unrecrystallized sites, and zero for recrystallized sites and particles. $H$ is the stored energy per site due to deformation, which is assumed to be uniform over the initially unrecrystallized material. Recrystallization occurs by a nucleation and growth process. Nucleation is modelled by adding small ( 3 sites) embryos to the material at random positions at the beginning of the simulation (i.e. site saturated nucleation). These embryos have $S$ values that differ from those of all other grains and particles. If $H$ is too small, the embryos are sub-critical and shrink away. The value of $H$ required for embryo growth depends on its surroundings. If $H / J>2$, an isolated embryo is super-critical and can grow, thus becoming a new grain nucleus. If $H / H<2$, the embryo must be adjacent to an existing grain boundary in order to become a nucleus; its growth then occurs preferentially along the prior grain boundaries.

The present simulations are initialized by performing grain growth simulations under the conditions described in Refs $[10,11]$ for a period of $10^{3} \mathrm{MCS}$ 
after which the microstructure contains approximately 1000 grains with a mean grain area of approximately 40 sites. At this point, single site "particles" are randomly placed within the microstructure with area fractions, $f$, between 1 and $10 \%$. Also, at this time, 3 site embryos are added to the system in numbers varying from 100 to 1000 . Simulations were performed with values of the stored energy relative to the grain boundary energy, $H / J$, of $0.1,1.01$ and 2.01 . The simulations were typically run for $30,000 \mathrm{MCS}$ and each data point on the plots below represent averages over 5 simulations, where each simulation had a different initial microstructure. Certain simulations were performed with no initial microstructure to simulate a (deformed) single crystal. Some other simulations were run with an initial microstructure but with a single large recrystallization nucleus (new grain) imposed at time zero.

The significance of the ratio of stored energy to grain boundary energy $(H / J)$ in relation to the initial grain size is as follows. The earlier simulation studies of recrystallization $[6,7]$ showed that the growth rate of new grains is critically dependent on $H / J$ and the prior grain structure. When $H / J<2$ new grains can only grow along the prior grain boundaries: for $H / J>2$, however, the new grains can grow in the absence of prior grain boundaries. As $H / J$ increases towards 5 , the growth rate increases towards a saturation value because, at high $H / J$, any site at the recrystallization front can switch from unrecrystallized to recrystallized. For $H / J>6$, spontaneous nucleation occurs throughout the lattice. All this implies that only a limited range of $H / J$ can be explored in the current Monte Carlo model.

The relationship between the $H / J$ values employed here and physical systems can be analyzed as follows. Physically, the stored energy per unit volume ( $\left.P^{\text {physical }}\right)$ is simply defined by the dislocation density that is removed on recrystallization (of order stress ${ }^{2}$ over modulus, see above), so $P$ is of order $10 \mathrm{MPa}$. The boundary energy ( $\left.B^{\text {physical }}\right)$ per unit volume is simply the grain boundary energy $(\gamma)$ divided by the grain size $\left(d^{\text {physical }}\right), B=\gamma / d^{\text {physical }}$. The ratio of these two energy densities is

$$
\frac{P}{B}=\frac{P d^{\text {physical }}}{\gamma}
$$

The equivalent considerations in the Monte Carlo model show that $P^{\text {model }}$ is the stored energy per point, divided by the area (volume) associated with that point, so $P^{\text {model }}=H / 2 \cdot 6 s^{2}$, where $s$ is the lattice parameter. Similarly the boundary energy density is given by $B=J /\left(s \cdot d^{\text {model }}\right) ; J / s$ is the boundary energy per unit length of boundary. So we get

$$
\frac{P^{\text {model }}}{B^{\text {model }}}=\frac{H s d^{\text {model }}}{2 \cdot 6 s^{2} J} \text {. }
$$

We know that typical values in simulation are $d^{\text {model }} \sim 6 s$, and $H / J=2$, so, equating the ratios of energy densities in the model and in experiment, we get

$$
\frac{P^{\text {physical }} d^{\text {physical }}}{\gamma}=\frac{6 H s^{2}}{2 \cdot 6 s^{2} J} \sim 6 .
$$

Rearranging and taking $\gamma=0.5\left(\mathrm{~J} \cdot \mathrm{m}^{-2}\right), P^{\text {physical }}$ $=10(\mathrm{MPa})$, we get

$$
d^{\text {physical }}=\frac{3}{10}=0.3 \mu \mathrm{m} \text {. }
$$

This analysis shows that the simulations correspond to a very fine grain size, though not one that is necessarily unphysical, especially if the initial microstructure is considered to represent the subgrain structure. In this sense, the simulations actually address the nucleation stage of recrystallization, rather than the later stages of growth. Realistic models of nucleation, however, would have to include the variations of mobility and boundary energy that are known to occur. The results of simulation of abnormal grain growth due to variable boundary mobility [14], for example, appear to be applicable to the growth of subgrains into recrystallization nuclei. Returning to the present simulations, a more appropriate scaling of grain sizes between simulation and physical experiment could be made by using lattice sizes ten times greater than presently employed. This would, however, be difficult and expensive to carry out on current computers because the number of lattice points would then be of order $10^{6}$ (instead of $\left.10^{4}\right)$.

The aim of this paper has been to simulate the effect of particles on recrystallization. An additional result of this work, however, has been to demonstrate the importance of grain growth both before and after recrystallization is complete. A thorough investigation of the interaction between grain growth from a finite initial grain size and randomly distributed particles has been performed and is in preparation for publication [34]. Preliminary results of that study have already been presented however [16]. It was found that some grain growth always occurs and that the correlation between boundaries and particles rises rapidly from that predicted for a random correlation. The same process can also be seen in the present recrystallization simulations, as described below, in both the deformed grains and in the recrystallized grains after recrystallization is complete.

\section{RESULTS}

In order to test the validity of the simulation procedure and to examine one of the simplest cases of primary recrystallization in the presence of second phase particles, a series of simulations were performed in which a relatively large, circular, recrystallized grain (area $\cong 80$ sites) was introduced into a matrix of unrecrystallized grains (mean area $\cong 40$ sites). Figure 1 shows the evolution with time of the resultant microstructure for a particle fraction of $2.5 \%$ and $H / J=1.01$. For this stored energy value, 

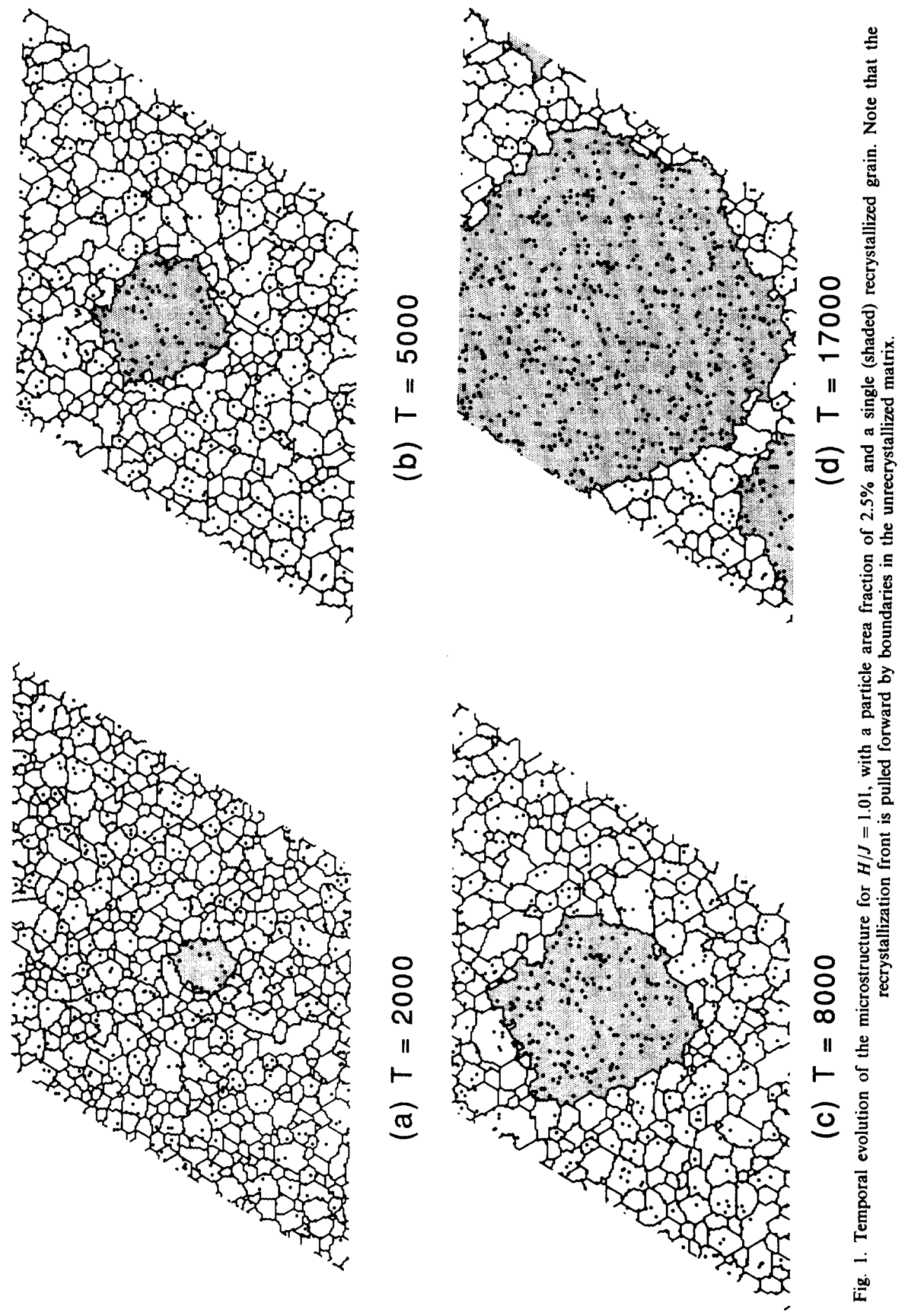


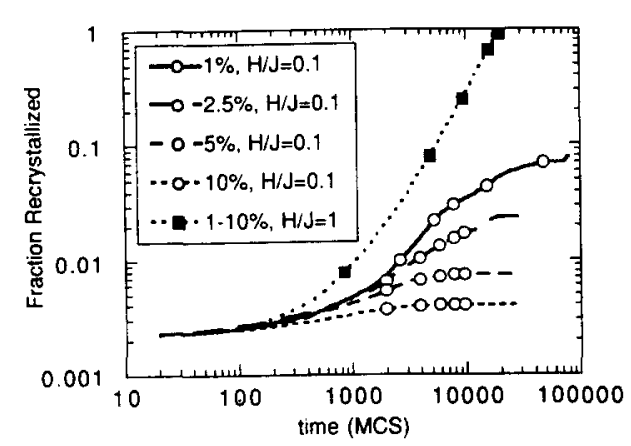

Fig. 2. Mean recrystallized grain area vs time for simulations with a single recrystallized grain and particle area fractions of $1,2.5,5$ and $10 \%$. Solid symbols denote $H / J=1.01$, showing no dependence on the particle fraction. Open symbols denote $H / J=0.1$, showing pinning of the recrystallized grain at high fractions and unpinned growth at small fractions.

the recrystallized grain grows and consumes the surrounding unrecrystallized matrix. The growing grain is clearly influenced by the microstructure and by particles on its periphery as is evident from the irregularity of the grain boundary separating the recrystallized and unrecrystallized regions. An analysis of the growth kinetics shows that the growth rate of the recrystallized grain increases with time until it reaches a steady state in which its area grows as time squared $\left(t^{2}\right)$ (see Fig. 2). This result is essentially the same as when no particles are present, suggesting that the particles do not impede significantly the progress of the recrystallization front. The recrystallization front is clearly influenced by the presence of the matrix grains which alter the curvature of the recrystallization front. That is, the centers of curvature of each segment of the recrystallization front lie outside the recrystallized grain. This curvature, together with the stored energy, provides the driving force for recrystallization growth.

In contrast to the case of $H / J=1$, where the recrystallized grain grows rapidly into the matrix, simulations for small $H / J(H / J=0.1)$ show that the growth of the recrystallized grain is effectively impeded by the presence of the particle dispersion (see Figs 2 and 3). Although the recrystallized grain size does initially increase with time, its growth appears to saturate at an area that depends on the particle density, as shown in Fig. 2. The important exception to these statements is that for the smallest fraction of particles, the growing grain never quite stops growing. A previous study [7] showed that a stored energy of 0.1 is sufficient for recrystallization to occur in these 2-D models. Therefore it is reasonable that at some sufficiently low particle fraction, recrystallization can go to completion. This is discussed further below.

Figure 4 shows two successive microstructures for a higher stored energy, $H / J=2.01$, in which growth occurs from the imposed circular grain at $t=0$ to a larger size at $t=1600 \mathrm{MCS}$. In this case the recrystal- lized front has a more irregular pattern, expected at this larger driving force [6], and has centers of curvature lying both inside and outside the new grain. In the absence of prior grain boundaries, Fig. 5 , at the same values of $H / J$ and $f$ as in Fig. 4 , the center of curvature lies, on average, inside the new grain. However the irregularity due to the statistical nature of the site switching operation [6] somewhat obscures the clarity of the general result. The important role of the matrix grain boundaries is that they provide an additional driving force for growth of the new grain as is readily seen by noting the times needed for the new grain to grow to the size shown in Figs $4(\mathrm{~b})$ and $5, t=1600 \mathrm{MCS}$ in the polycrystalline matrix and $t=2800 \mathrm{MCS}$ in the single crystal respectively. Recrystallization is known to be inhibited in a single crystal matrix for $H / J<2$ even in the absence of particles $[6,7]$. Simulations of recrystallization with particles but no grain structure (single crystal) at $H / J<2$ confirmed that this inhibition still occurs.

Figure 6 shows the density of particles on the migrating recrystallization front as a function of time for the single crystal simulation shown in Fig. 5. The interfacial density of particles is defined as the ratio of the number of particles on the interface to the total number of lattice sites on the interface. The critical result is that, although the interfacial particle density fluctuates statistically because of the limited number of sites on the recrystallization front, the mean value is close to the (Zener) random value of $f(2.5 \%)$. This is the result previously reported experimentally for recrystallization into single crystals by Ashby et al. [21], and is of course different from the much higher than random correlation between particles and grain boundaries seen in simulations of grain growth $[11,16,17,19]$.

Many of the basic results of the growth of the large, isolated recrystallized grain carry over to the more realistic simulations which include spatial distributions of small recrystallization nuclei. Figure 7 shows the density of particles on both the recrystallization front, that is on boundaries between new and old grains only, and on all grain boundaries for $H / J=1.01$ and $f=5 \%$. Figure 7(a) shows the results for 200 embryos while 7 (b) shows the results with only 100 embryos. Again the number density of particles on the recrystallization front fluctuates about the random value, $f$, of $5 \%$. The density on all boundaries starts very close to the random value but rapidly increases as the matrix grains grow and become pinned at particles, see Fig. 4(a). A similar increase in density of particles on boundaries has been reported for grain growth from identical starting microstructures of finite grain size $(\langle A\rangle \sim 40)$ but withoug any stored energy or recrystallization $[16,34]$. The surprisingly low particle density values at zero times are a consequence of the fact that the embryos are inserted into the microstructure after the particles. The embryos are not permitted to overlap 

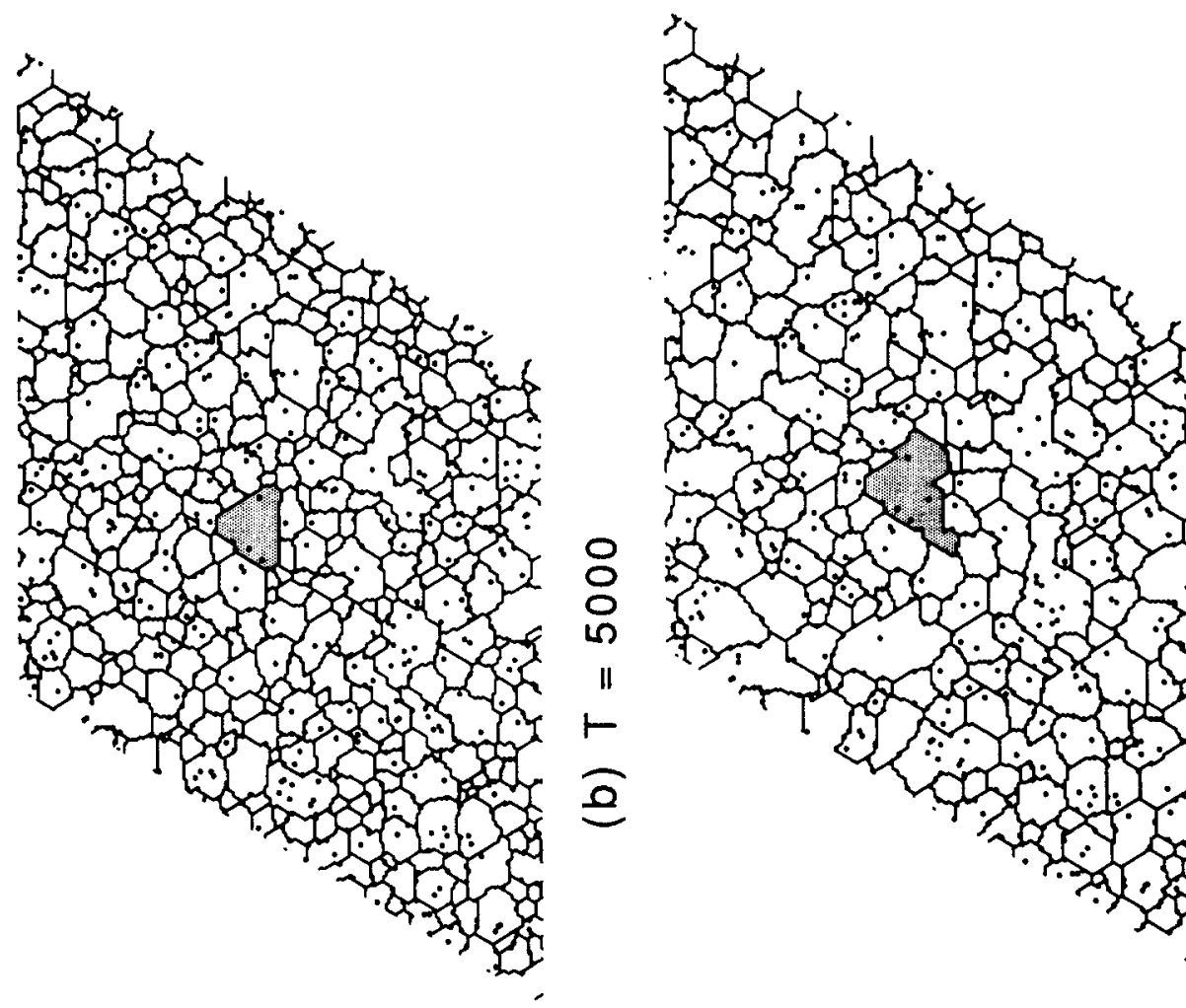

क⿺辶.
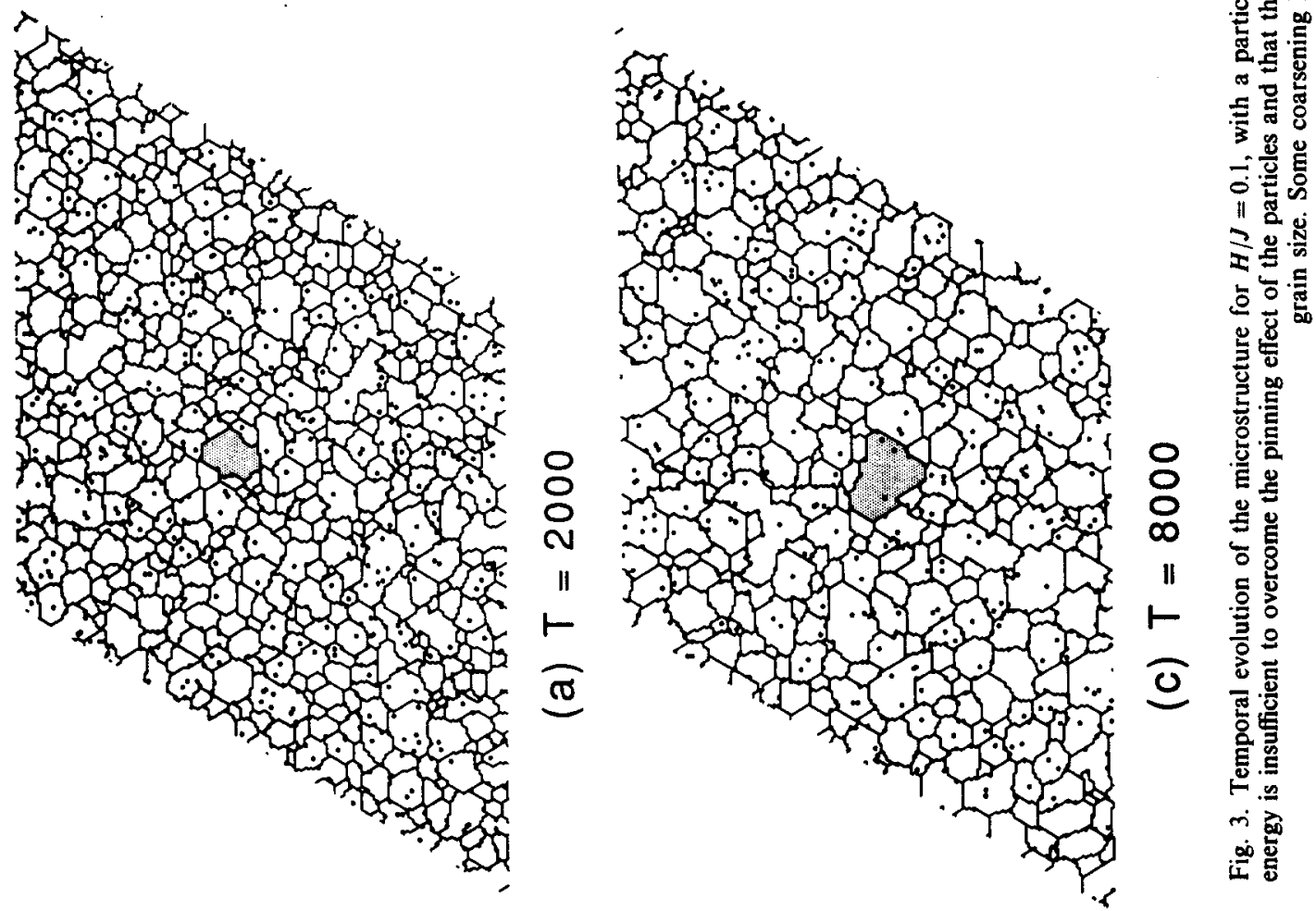


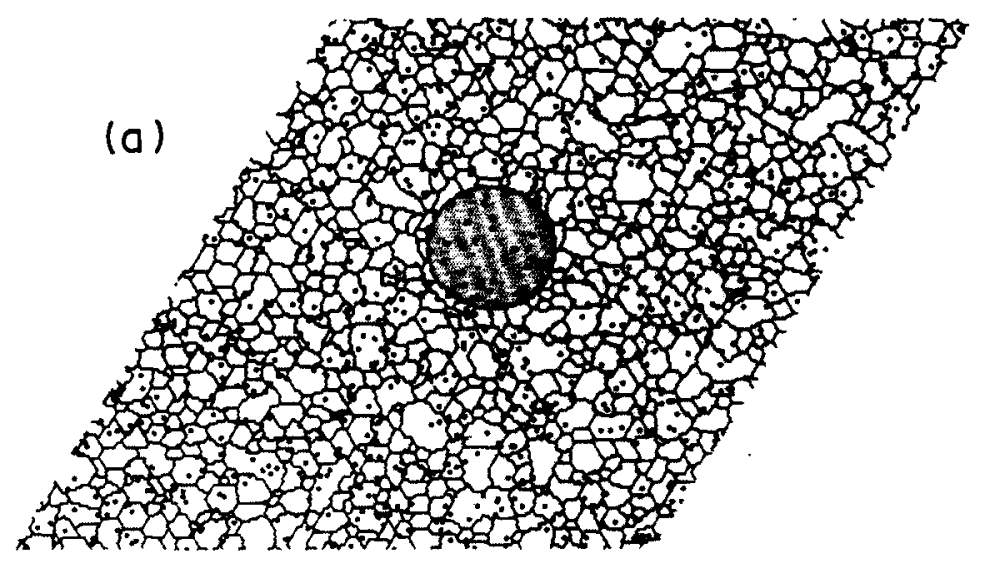

$$
T=0
$$

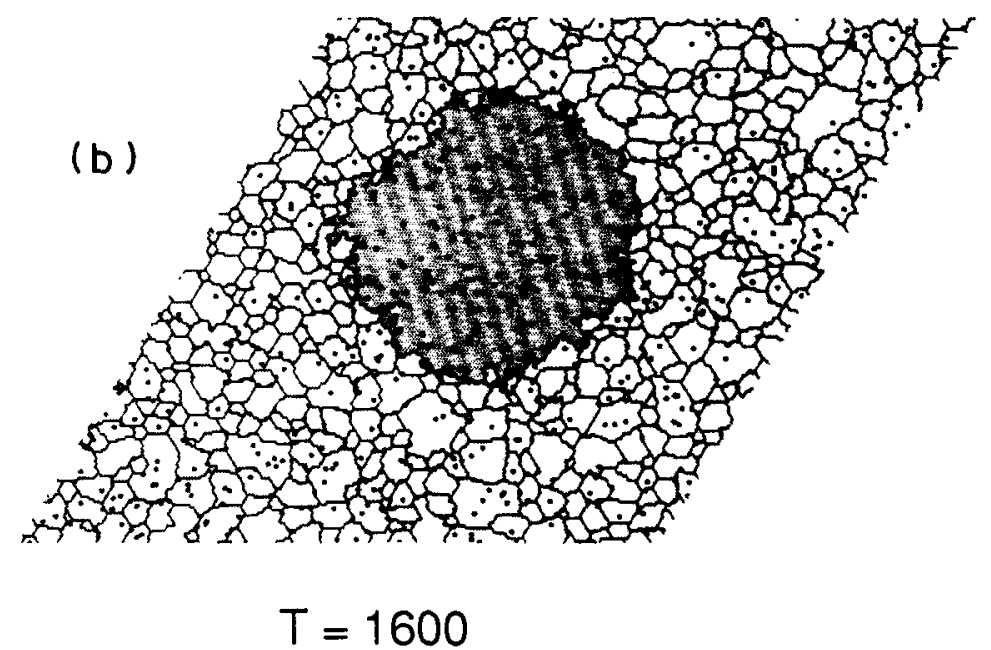

Fig. 4. Temporal evolution of the microstructure for $H / J=2.01$, with a particle area fraction of $2.5 \%$ and a single (shaded) recrystallized grain. Note that some portions of the recrystallization front have advanced beyond those regions which are held back by particles.

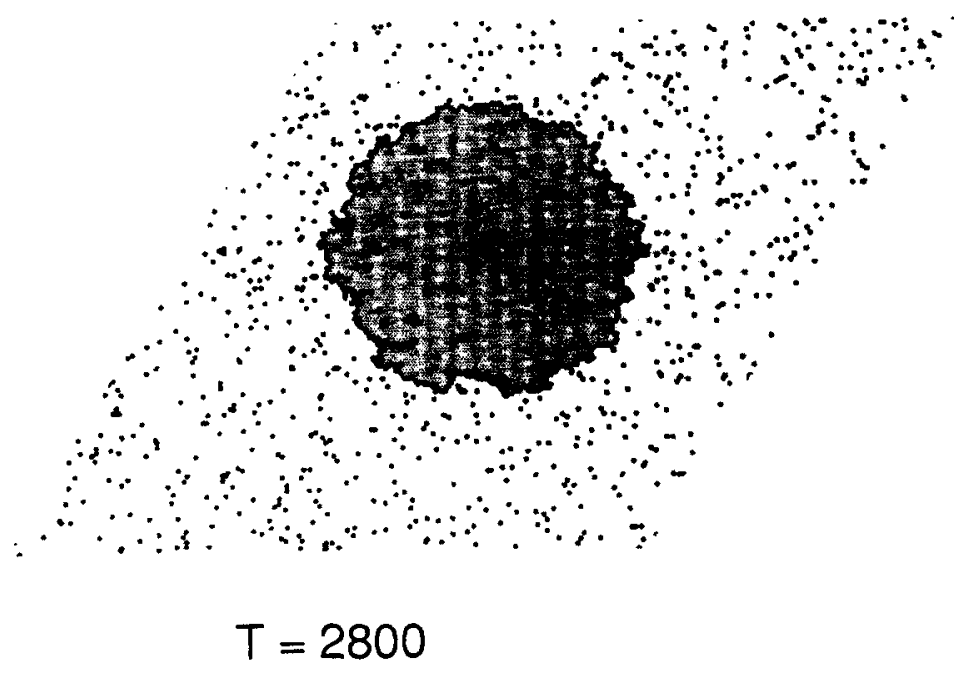

Fig. 5. A single (shaded) recrystallized grain growing into a single crystal matrix with a particle area fraction of $2.5 \%$ and $H / J=2.01$. Note that the growth of the recrystallized grain is slower than in Fig. 4 where the unrecrystallized region is polycrystalline. 


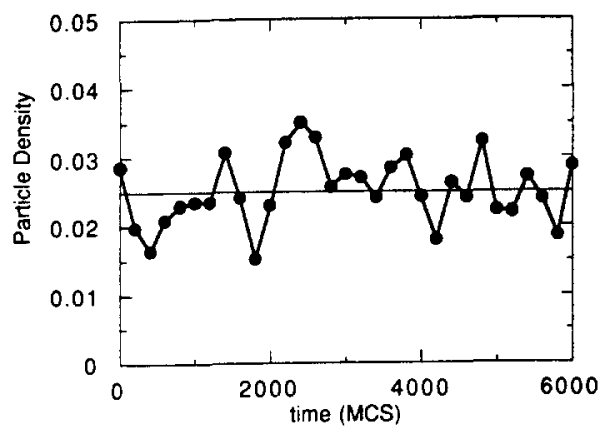

Fig. 6. Plot of the fraction of particles on a recrystallization front for $H / J=2.01$, with a particle area fraction of $2.5 \%$ and a single recrystallized grain and no prior grain structure. The line at fraction $=0.25$ indicates the value expected for random intersections of particles with boundaries.

with particles so that a significant fraction of the recrystallization front sites are excluded from having particles, that is to say, none of the 3 sites inside the embryo (25\% of the boundary sites associated with each embryo) can be a particle. The density shows a plateau when the structure is partially recrystallized but it rises towards the end of recrystallization when the only boundaries remaining are those between new grains since all the old deformed grains have been consumed. The recrystallization boundaries, between old and new grains, with a random density of particles are, of course, then disappearing by impingement.

Figure 8 shows the temporal evolution of the microstructure for the simulation just described, Fig. 7(a), that is for $H / J=1.01, f=5 \%$ and 200 embryos. The recrystallized grains grow into the unrecrystallized matrix with the same interface morphology as observed in the simple case shown in Fig. 1; that is with their centers of curvature outside the recrystallizing grains. Unlike the single recrystallized grain case, however, the individual recrystallized grains do not grow to such large sizes due to their mutual impingement. The time dependence of the fraction of the sample which has recrystallized, $F$, is shown as a function of time for three different values of the stored energy, Fig. 9(a). As in the simple test case described above, when $H / J \geqslant 1$ the recrystallized grains grow rapidly and complete recrystallization $(F=1)$ is achieved at relatively early times $(t<5000 \mathrm{MCS}$, at $H / J=1.01$ and $t<2000 \mathrm{MCS}$ at $H / J=2.01$ ). On the other hand, when $H / J$ is only 0.1 the recrystallized fraction quickly saturates at a low value and remains unchanged over very long times, i.e. the recrystallized grains are successfully pinned by the particles.

Further analysis of the growth kinetics shows that for both the $H / J=1.01$ and 2.01 cases, an Avrami exponent of 2 is obtained for much of the reaction [Fig. 9(b)]. This result is consistent with that obtained in the absence of a second phase dispersion. The initial small slope in the Avrami plot is attributable to a combination of the finite initial volume fraction recrystallized at time zero and the loss of the finite size, sub-critical embryos. This is most apparent for the lowest $H / J, H / J=0.1$, for which the initial slope is negative. A plot of the logarithm of the recrystallized grain area $\langle A\rangle_{\text {Rex }}$ vs time [Fig. 9(c)] shows that the recrystallized grain size saturates at long times. For small $H / J$, this is due to particles restricting the growth of the recrystallized grains into the unrecrystallized matrix. For large stored energy, however, the recrystallized grain size does not saturate until recrystallization is complete. For the cases depicted in Fig. 9, the grain size at the end of recrystallization is larger than would result if the grain size were determined by grain growth (in the absence of recrystallization) in the presence of a particle dispersion. Therefore, by the time recrystallization is complete, normal grain seems to be entirely inhibited. In this way, the final microstructure is simply a picture of the system at the end of recrystallization and further annealing has essentially no effect on the microstructure (assuming no particle coarsening or dissolution). This behavior can explain why the final grain size for $H / J=2.01$ is smaller than that for $H / J=1.01$. At larger values of $H / J$, more of the recrystallization nuclei (embryos) added at the beginning of the simulation are supercritical than at lower stored energies. This was verified by examining the number
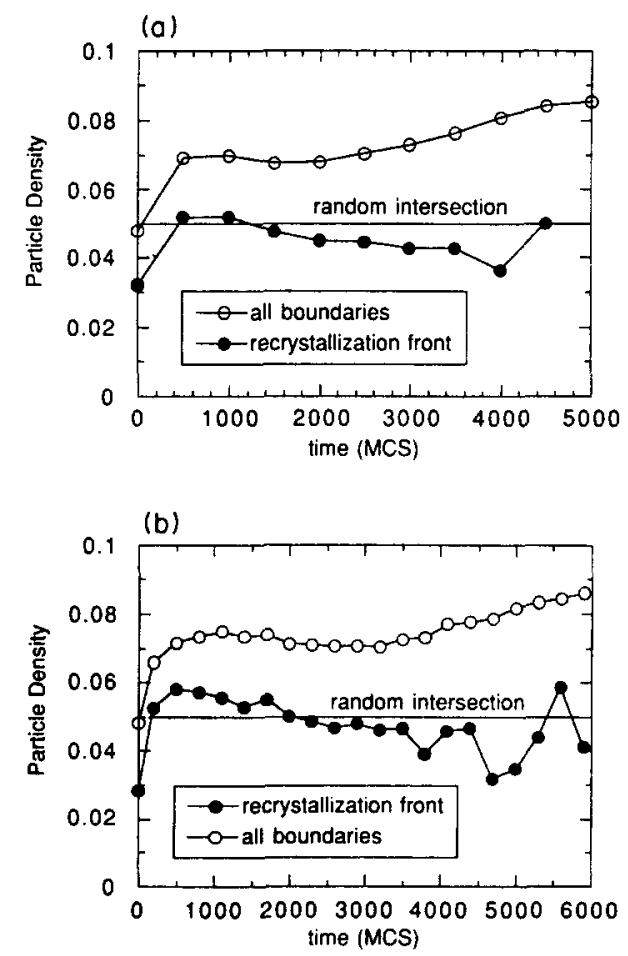

Fig. 7. Plot of the fraction of particles on boundaries for $H / J=1.01$, with a particle area fraction of $5 \%$ and two different nucleation densities, (a) 100 and (b) 1000 site saturated nuclei. The solid symbols represent particles on all boundaries, the open symbols represent particles on the recrystallization front and the constant horizontal line indicates the value expected for random boundary-particle intersections. 

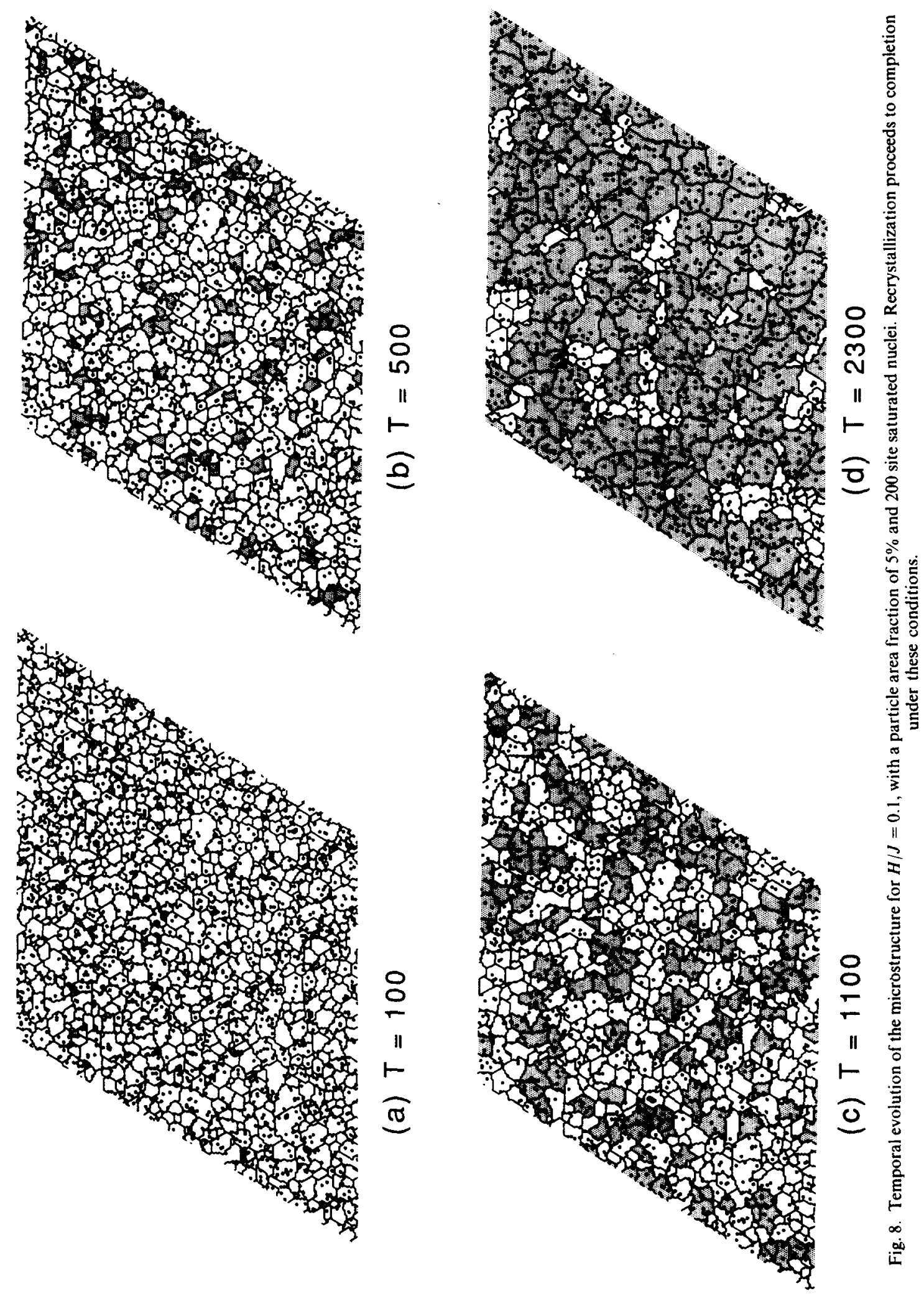

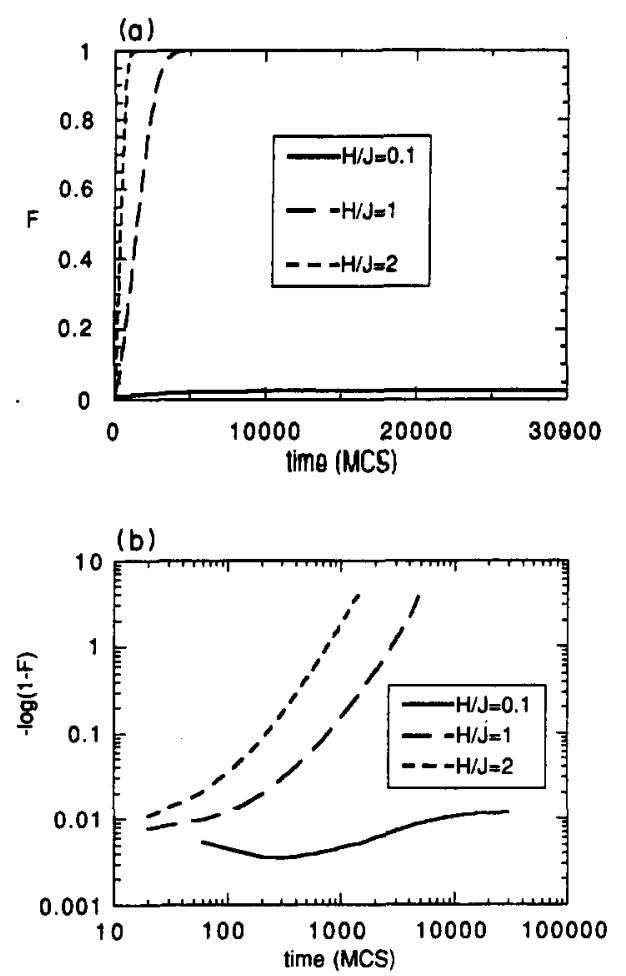

(c)

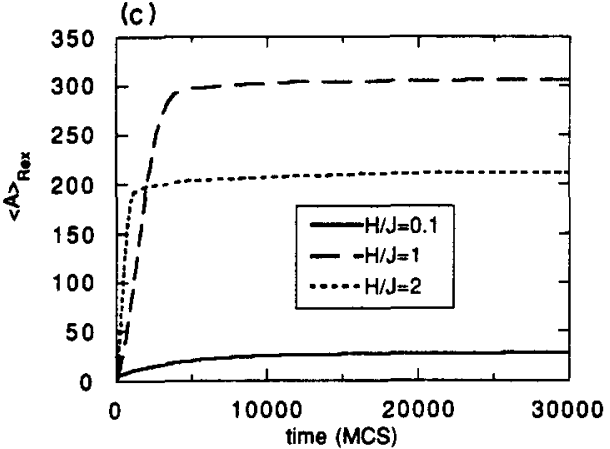

Fig. 9. (a) Plot of fraction recrystallized, $F$ vs time for $H / J=0.1,1.01$ and 2.01 , with a particle area fraction of $5 \%$ and 200 site saturated nuclei. (b) Johnson-Mehl-AvramiKolmogorov (JMAK) plot for the same conditions as in (a). The initial decrease in fraction recrystallized for $H / J=0.1$ is due to the disappearance of sub-critical nuclei. (c) Plot of mean recrystallized grain area vs time. The particle fraction is high enough to pin the microstructure after completion of recrystallization. The origin of the crossing of the curves for $H / J=1.01$ and 2.01 may be traced to the fact that fewer embryos are supercritical at $H / J=1.01$.

of recrystallized grains as a function of time. Therefore, since growth competition between recrystallized grains due to grain boundary curvature effects is negligible, the final recrystallized grain size is a measure

only of the inverse density of supercritical nuclei.

The effect of different particle fractions at fixed stored energy is shown in Fig. 10 where we plot the recrystallized fraction [10(a)], the JMAK plot [10(b)] and the mean recrystallized grain area as a function of time $[10(c)]$, respectively, for $H / J=1.01,200$ embryos and particle fractions between 0 and $10 \%$.
Clearly, at this value of stored energy, the particles have a negligible effect on the recrystallization behavior, as demonstrated by the nearly identical curves for recrystallization with no particles and for high particle densities. The only noticeable effect of variations in particle density at this higher stored energy is that more embryos survive at higher particle densities because of heterogeneous nucleation. One consequence of this is that the kinetics are slighlty accelerated. The second is that the larger the particle density, the smaller is the final recrystallized grain size. This small effect occurs because the particles provide additional heterogeneous nucleation sites. At a stored energy of 1.01 , a nucleus can only survive if it
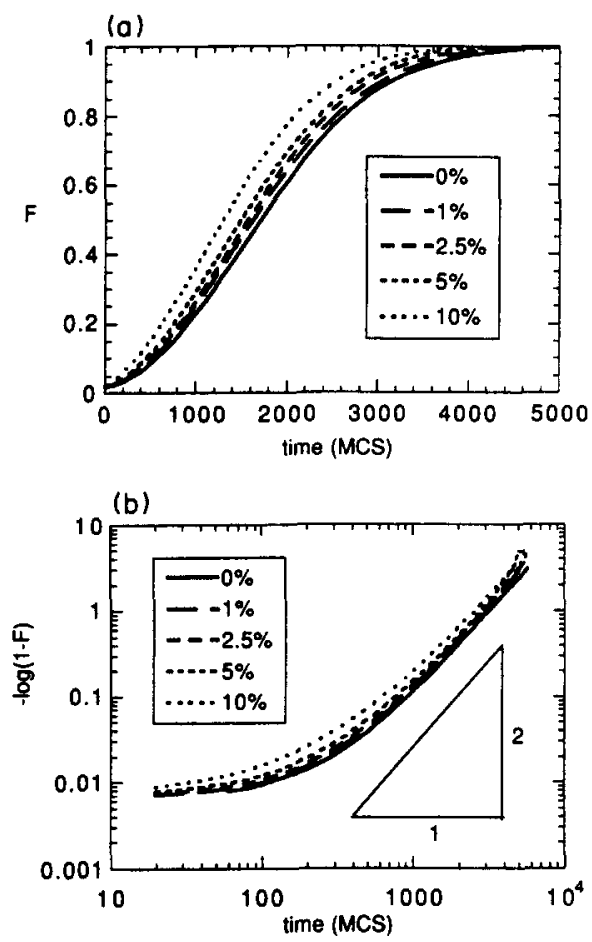

(c)

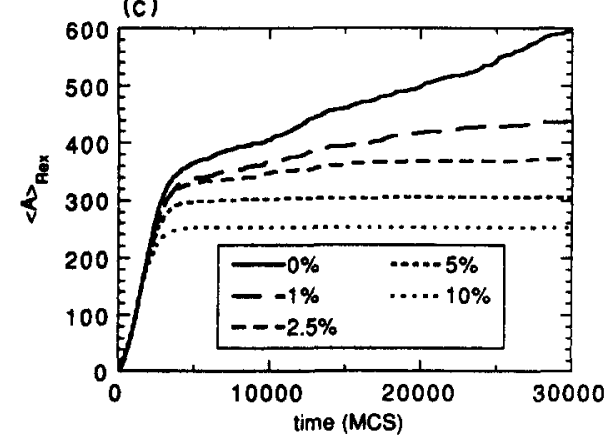

Fig. 10. (a) Plot of fraction recrystallized vs time for $H / J=1.01,200$ site saturated nuclei and particle area fractions of $0,1,2.5,5$ and $10 \%$. (b) The JMAK plot for the same conditions as in (a). In both plots, note the very minor dependence on particle fraction. (c) Plot of mean recrystallized grain area vs time. The separation of the curves at long times is the result of (i) more nuclei being super-critical at higher particle fractions and (ii) grain growth after recrystallization at low fractions. 

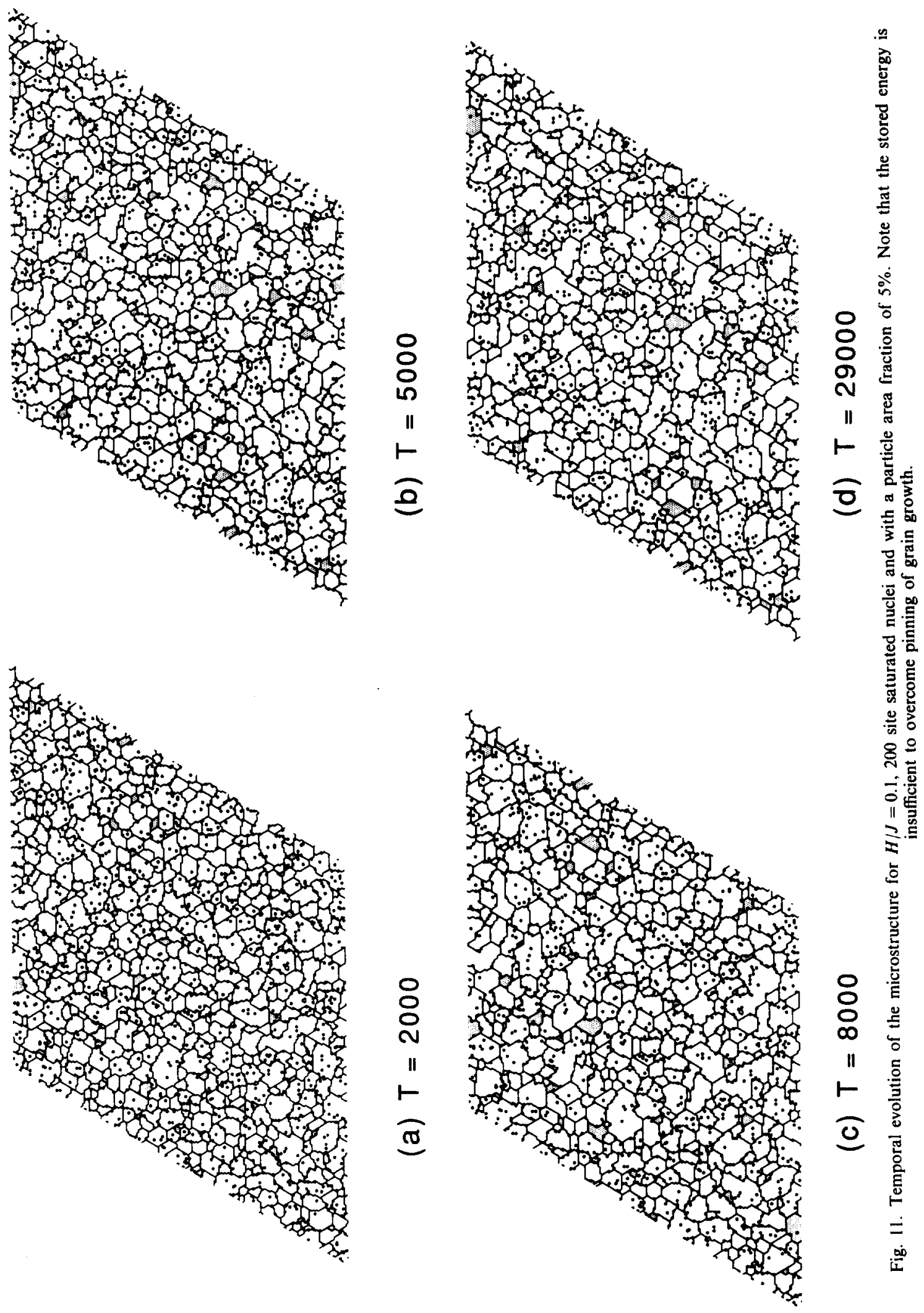

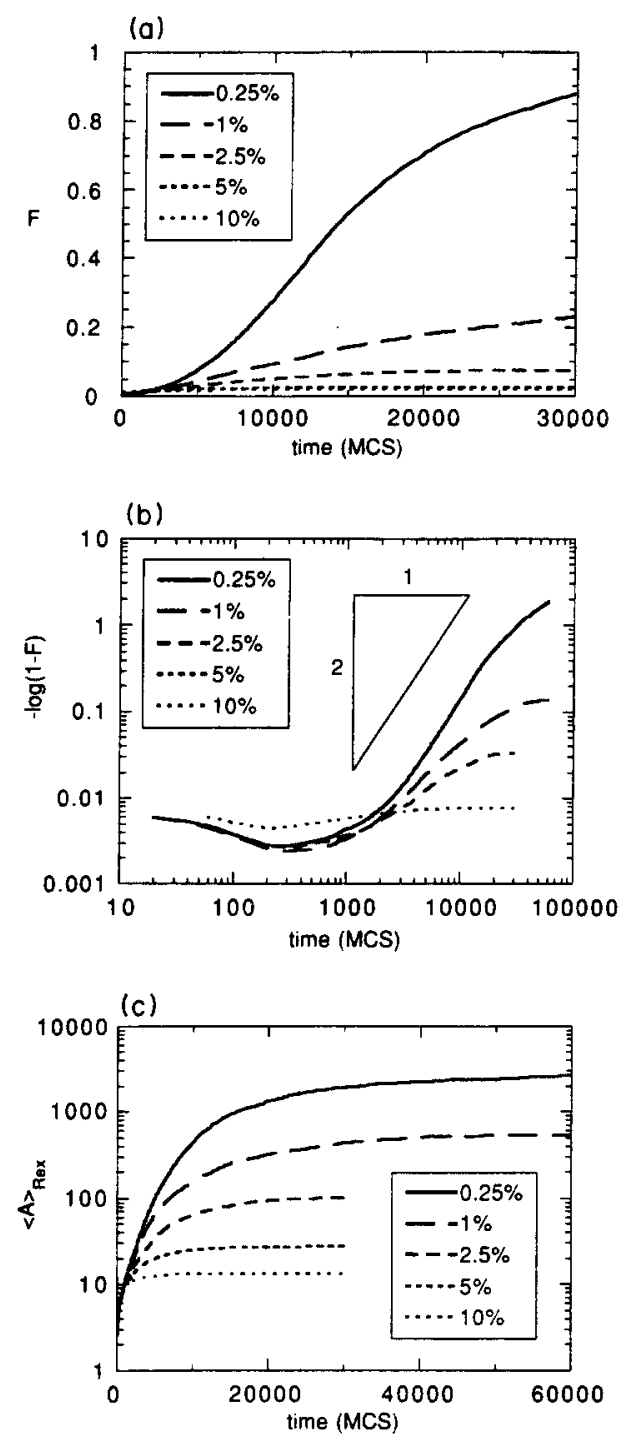

Fig. 12. (a) Fraction recrystallized vs time for $H / J=0.1$, 200 site saturated nuclei and particle area fractions of 0.25 , $1,2.5,5$ and $10 \%$. Note the strong dependence on particle fraction at low particle fractions. (b) The JMAK plot for the same conditions as in (a). (c) Logarithmic plot of the mean recrystallized grain area versus time. The separation of the curves at long times is the result of particle pinning at high fractions and the completion of recrystallization at low fractions.

nucleates heterogeneously, i.e. on a boundary or particle [7]. Note that for the higher particle densities, no grain growth occurs after recrystallization. However for the smaller particle densities some grain growth does continue after recrystallization is complete.

The effects of variations in particle density at lower stored energies (where recrystallization does not go to completion) is much more pronounced. Figure 11 shows the temporal evolution of the microstructure for $H / J=0.1$ and a particle area fraction of $5 \%$. Some growth of the recrystallized grains beyond particles is clearly observed, but unlimited growth, as in Fig. 8 where the stored energy is larger $(H / J>1)$, does not occur. Figure 12 shows the time dependence of the recrystallized fraction [12(a)], the JMAK plot [12(b)] and the mean recrystallized grain area [12(c)], respectively, for $H / J=0.1$ and particle area fractions between 0.25 and $10 \%$. The initial negative slope in the JMAK plots, Fig. 12(b), due to the loss of sub-critical embryos, is smallest for the highest particle fraction because of heterogeneous nucleation on particles. For the higher particle fractions, both the final recrystallized fraction and the recrystallized grain size saturate at long times at values that decrease with increasing particle concentration. For the lowest fraction $(0.25 \%)$, however, recrystallization goes essentially to completion, albeit rather slowly compared to the simulations with higher stored energy. Analysis of the JMAK plot, Fig. 12(b) shows that the maximum slope is approximately 2 for small particle fractions where recrystallization goes to completion, but decreases with increasing particle density when it does not. The pinned partially recrystallized grain area appears to scale with $f$ as $1 / f^{*}$, where $\alpha \sim 0.5$, Fig. 13, which is a weaker dependence than that observed in grain growth $\left(\langle A\rangle_{\text {Rex }} \propto f^{-1}\right)^{11}$. This does not apply for the smallest particle fraction $(0.25 \%)$ where recrystallization goes to completion (denoted by a solid symbol in Fig. 13).

At large stored energies $(H / J=1.01)$ variations in the number of embryos added to the system at fixed particle fraction $(f=5 \%)$ have an effect both on the time for complete recrystallization to occur and on the final grain area. Figure 14 shows the time dependence of the recrystallized fraction [14(a)], JMAK plot $[14$ (b)] and the mean recrystallized grain area [14(c)], respectively, for $H / J=1.01$, a particle fraction of $5 \%$ and numbers of embryos between 100 and 1000. As expected, the time required for complete recrystallization increases as the number of nuclei is decreased. The time dependence of the mean recrystallized grain size is independent of the number of nuclei $(>0)$ at early times before the individual recrystallized grains impinge. At later times, where impingement becomes important, the curves corresponding to different numbers of nuclei grow to

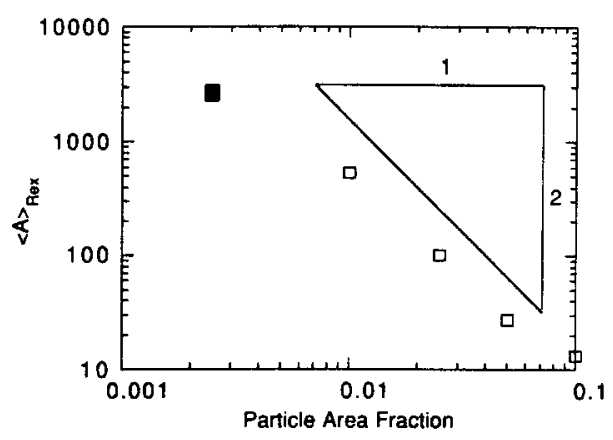

Fig. 13. Logarithmic plot of the mean recrystallized grain area at long times vs particle fraction, from the data in Fig. 12(c). The slope of the plot is approximately $-1 / 2$ except for the lowest particle fraction $(f=0.25 \%$, solid symbol) where recrystallization goes to completion. 

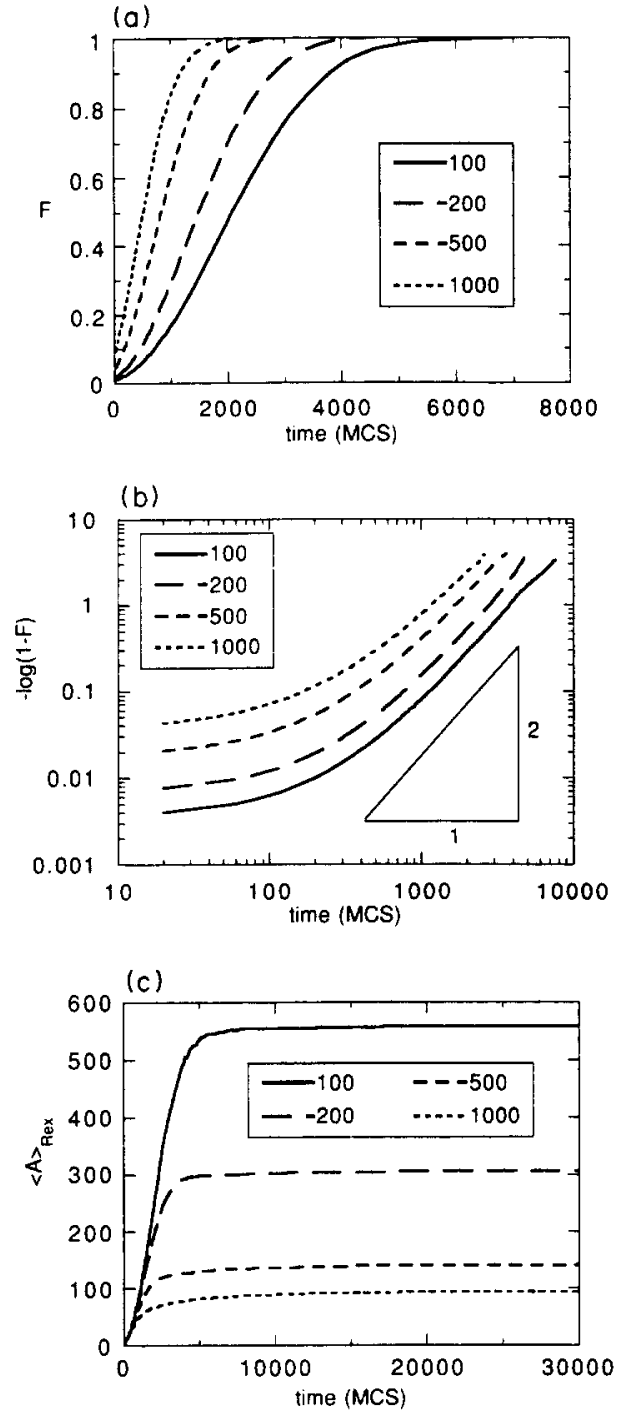

Fig. 14. (a) Plot of fraction recrystallized vs time for $H / J=1.01$, with a particle area fraction of $5 \%$, and 100 , 200,500 and 1000 site saturated nuciei (noted as an initial fraction recrystallized in the plot). (b) JMAK plot for the same conditions as in (a). The maximum slope approaches 2 as expected for site saturated nucleation and twodimensional growth. (c) Plot of the mean recrystallized grain area vs time.

different final grain sizes. For comparison, an additional simulation was performed with the identical number of particles but with no embryos added. Since no nuclei were present, only normal grain growth of the prior grains in the presence of a particle dispersion occurred and the final grain size was approximately 100 sites [see Fig. 14(c)] which has been shown [16] to be the case in pinned grain growth from a finite initial grain size of $\langle A\rangle=40$.

When the stored energy is small $(H / J=0.1)$, however, the fraction recrystallized, Fig. 15(a), is a function of the number of nuclei; the larger this number, the greater the final fraction recrystallized. This may be attributed to each super-critical embryo growing to a size that is limited by the particle density. For the lowest particle densities, however, continued growth occurs, as seen previously in Fig. 2 for the isolated recrystallized grain. The JMAK plot, Fig. 15(b), shows an initial decrease in the fraction recrystallized [not visible in the linear plot of Fig. 15(a)] that is the result of subcritical embryos shrinking away [7]. The mean size of the recrystallized grains, Fig. 15(c), is independent of the number of nuclei for the particle densities considered here $(f=5 \%)$. For this low value of the stored energy and at $f=5 \%$ the mean recrystallized grain size is less than the matrix grain size. However, at $f=0.25 \%$ the recrystallized grains grow much larger than the matrix grains, see Figs 12(c) and 15(c).

We now turn to a comparison of the microstructures in the final state; Fig. 16 shows the final microstructures for a stored energy of $H / J=0.1,200$
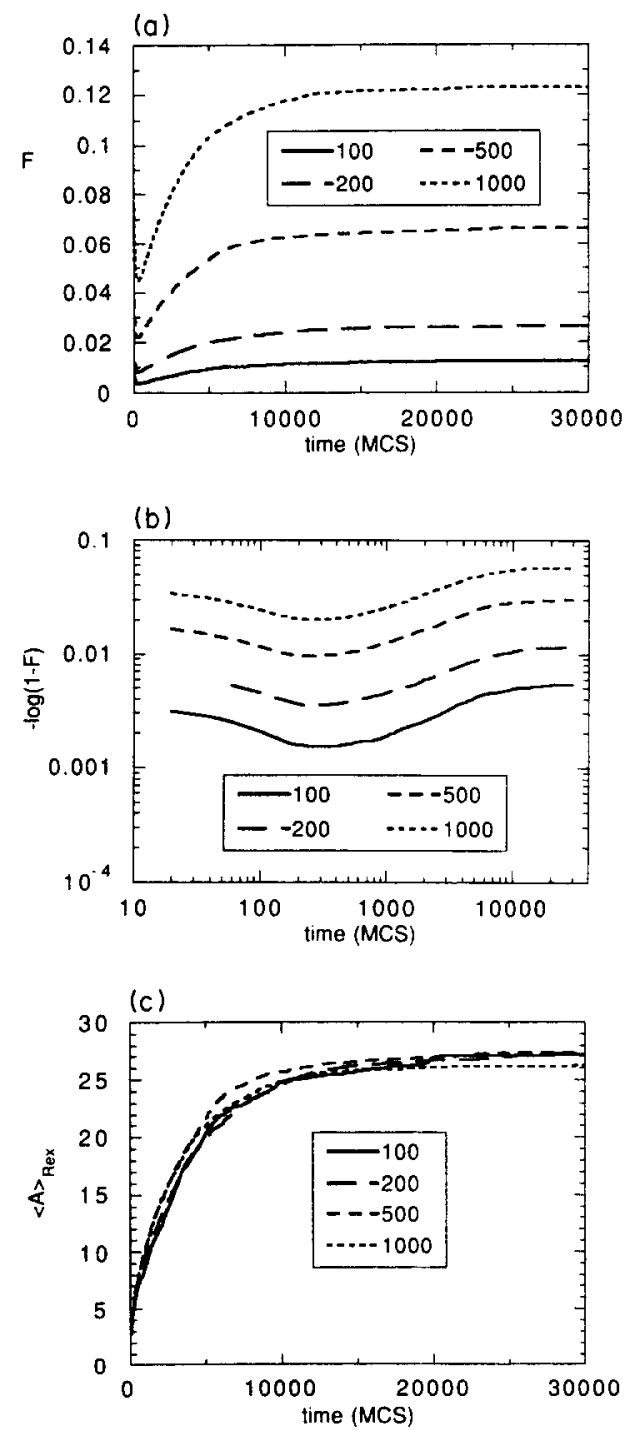

Fig. 15. (a) Plot of fraction recrystallized vs time for $H / J=0.1$, with a particle area fraction of $5 \%$. and 100,200 , 500 and 1000 site saturated nuclei. (b) JMAK plot for the same conditions as in (a). (c) Plot of the mean recrystallized grain area vs time. 
3
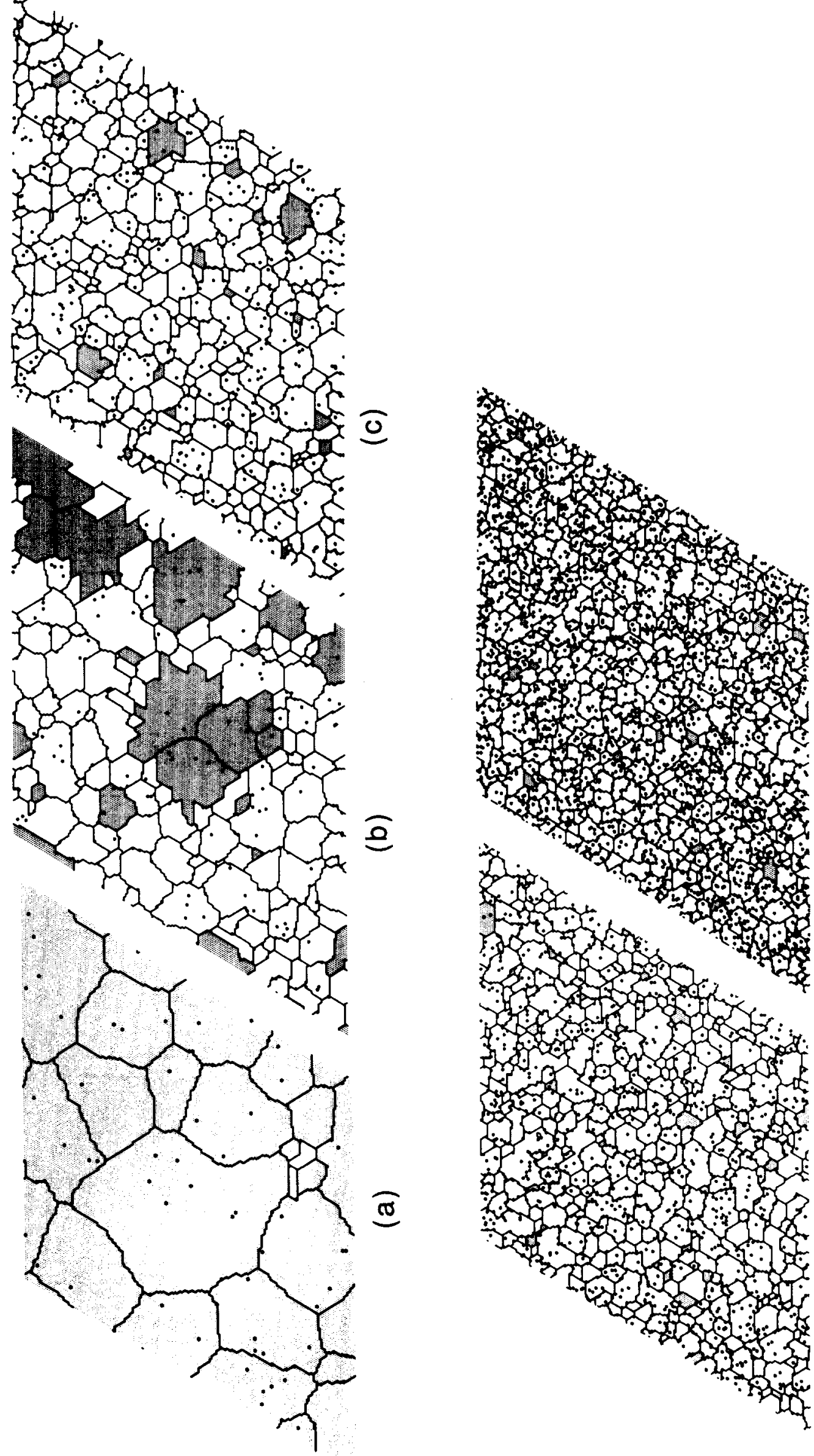

递

$\frac{1}{5}$

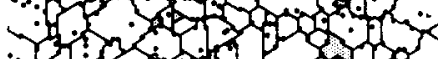

in

Ho

th E

ip

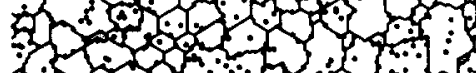

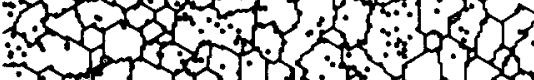
in 

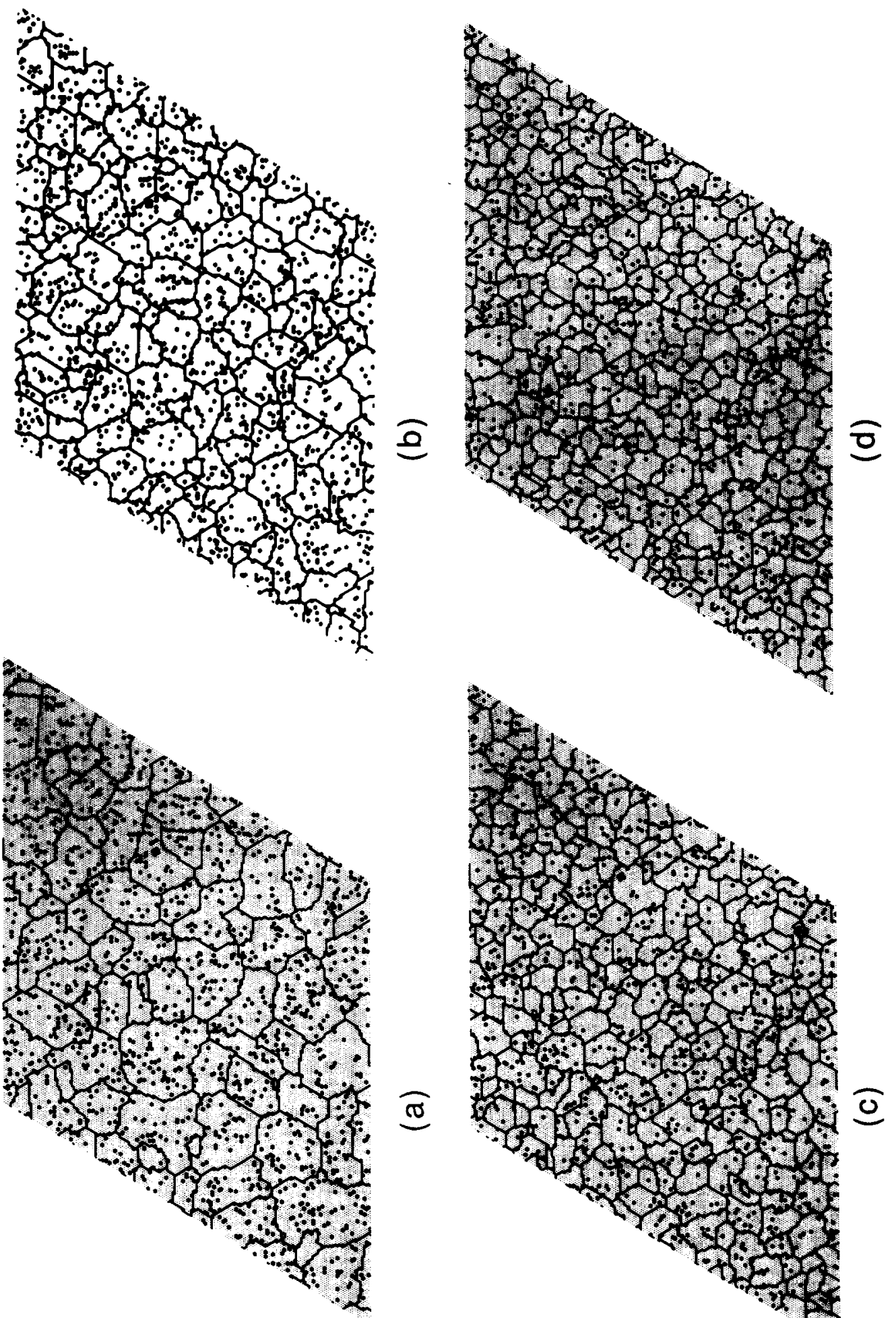

$\frac{5}{8}$
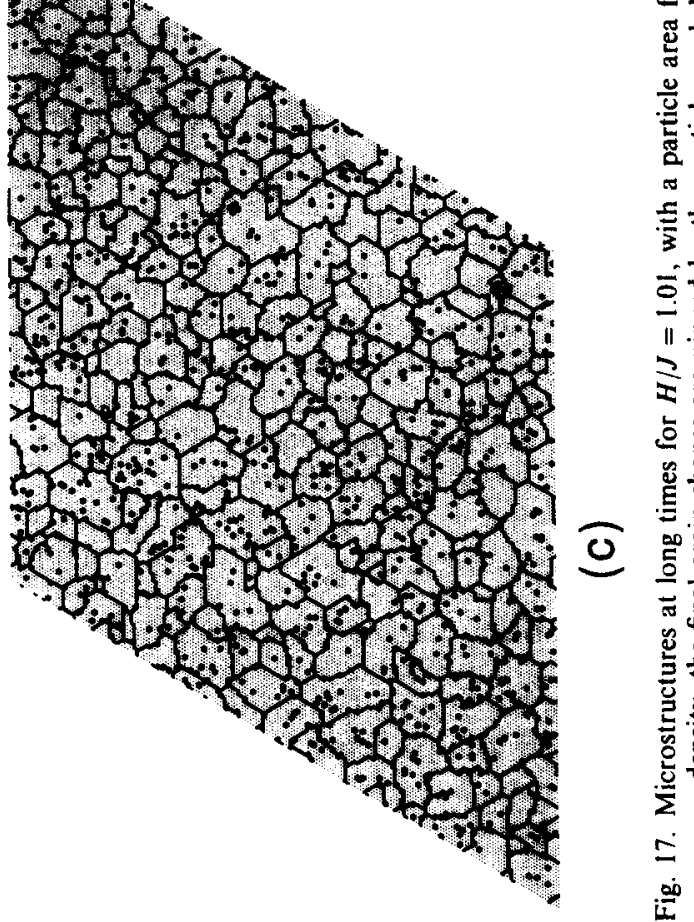
embryos at zero time and various volume fractions of particles. At this low stored energy, and when the recrystallization does not go to completion, there is little difference between these microstructures and those observed for normal grain growth in the presence of particles, other than a bais towards larger grains sizes for the recrystallized grains.

The final microstructures for particle fraction of $5 \%$ and various numbers of embryos at the larger stored energy of 1.01 , Fig. 17, show some interesting trends. For large numbers of embryos (small final grain size), the grain shapes are compact and similar to those observed in grain growth. This apparently occurs because the final grain size is small enough for some grain growth to occur after recrystallization. For small numbers of embryos (large final grain size), however, there are obvious departures from compact shapes. The elongated grain shapes observed for the small initial embryo density case reflect the spatially random nature of the nucleation process and they are preserved by the pinning action of the particles. When recrystallization occurs in the absence of particles [6], grain growth occurs concurrently with recrystallization and the non-compact shapes are eliminated. During recrystallization, however, the grain morphology is non-compact as was discussed in Ref. [6].

\section{DISCUSSION}

A major conclusion from the simulations at stored energies $H / J>1$ is that the particle density on the recrystallization boundaries is close to the Zener random value as found by Ashby et al. [21] in their single crystal experiments. This result means that the Zener drag in 2 dimensions [19] will be given by the random value

$$
Z_{\mathrm{R}}=\frac{3 f \gamma}{\pi r}
$$

and not by the higher value that applies when the boundaries are highly correlated with particles, as occurs during grain growth. The correlated value, $Z_{\mathrm{C}}$, is readily obtained as the product of a drag force per particle which, in 2 dimensions, is $2 \gamma$ [19], and the number per unit length of boundary $N_{1}$.

$$
N_{1}=\frac{1}{\Delta_{2}}=\frac{f^{1 / 2}}{\alpha r} .
$$

$\Delta_{2}$ is the mean nearest neighbor distance in 2 dimensions and $\alpha$ is a constant that is close to unity. This then yields the Zener drag for boundaries that are highly correlated with particles

$$
Z_{\mathrm{C}}=\frac{2 \gamma f^{1 / 2}}{\alpha r}
$$

This continuum calculation can be readily compared to the triangular lattice simulation by assigning each triangular lattice an area $a$, a radius $r=(a / \pi)^{1 / 2}$, and a side of length $s=0.62 a^{1 / 2}$ and an arbitrary thickness $t$. The stored energy $P$ is $H / a t$ and the boundary energy $\gamma$ is $J / s t$. Combining these values gives the stored energy $P$ just equal to $Z_{\mathrm{R}}$ when

$$
H / J=1.8 f \text { (random intersections). }
$$

The stored energy is just equal to $Z_{\mathrm{c}}$ when

$$
H / J=5.7 f^{1 / 2} \quad \text { (correlated intersections). }
$$

For the range of particle fractions used here, $f=0.0025$ to 0.1 , this gives, for random intersections, $H / J=0.0045$ to 0.18 [equation (13)]. For the stored energies where random intersections were observed, $H / J>1$, it is clear that the stored energy is large enough that a migrating recrystallization front sweeps past any particles it encounters with no delay. This is an agreement with the random intersections found by the simulations at this value of stored energy. The critical values of $H / J$ for recrystallization with correlated intersections, again for $f=0.0025$ to 0.1 , are, from equation (13), $H / J=0.285$ to 1.8 . These higher values indicate the much stronger drag expected when boundaries are strongly correlated with particles and suggest that, if such strong correlation ever developed, recrystallization should be strongly inhibited.

These continuum calculations, though interesting, are not strictly appropriate to analyzing the local conditions at the intersection of a particle and a migrating grain boundary in the present two-dimensional simulations. The situation in the discretized Monte Carlo model is illustrated by Fig. 18 as a portion of the lattice in which a recrystallizing grain is indicated by $R$ at the center of each lattice point, the deformed grain by $\mathbf{D}$ and a particle by $\mathbf{P}$. The solid irregular lines represent grain boundaries each of energy $J$, and the light paired lines represent zero energy bonds within grains. The stored energy per site, $H$, is released when $\mathrm{D}$ transforms to $(\rightarrow) \mathrm{R}$. The energy change for the switching operation, $D_{1} \rightarrow R$, is then

$$
\Delta E^{(\mathrm{D} \mid \rightarrow \mathrm{R})}=-3 J+3 J-H=-H .
$$

So for all finite values of $H / J>0$ the switch will occur and will not be reversed. The next step, $D_{2} \rightarrow R$ is more difficult however and gives an energy change of

$$
\Delta E^{(\mathrm{D} 2 \rightarrow \mathrm{R})}=-3 J+2 J-H=J-H .
$$

This switch can only occur, irreversibly, for $H / J>1$ and cannot occur for $H / J<1$ (for simulations at $0 \mathrm{~K}$ ). The same situation arises for a step arriving from the right (i.e. after $D_{4} \rightarrow R$ ) $D_{3}$ cannot go to $R$ until $D_{2}$ has switched. Continued arrival of steps from left and right cannot alter this situation of a sharp vertex of the deformed grain D pinned at the particle $P$. This is the situation seen in normal grain growth $(H / J=0)$ [11], when curvature of a shrinking grain is removed by replacing it by a vertex pinned by a particle.

The boundary can only escape from the particle by an extra step coming down the boundary to allow $D_{6}$ or $D_{8}$ to switch, which then allows $D_{2}$ or $D_{3}$ to switch. 
(a)

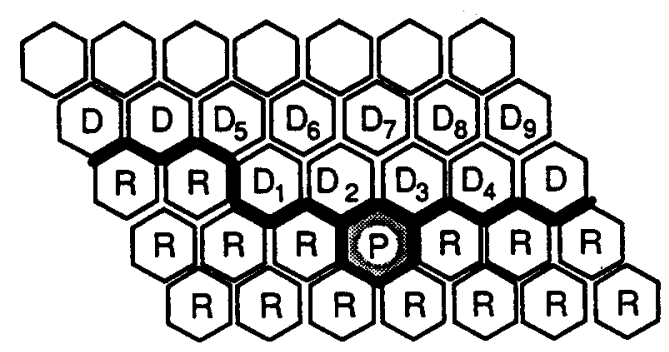

(b)

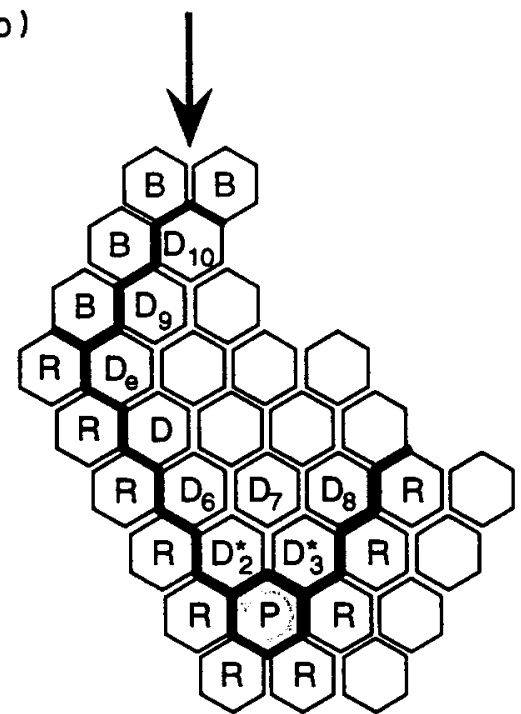

Fig. 18. Schematic diagrams of the configuration of sites in the model in the vicinity of an intersection of the recrystallization front and a particle. The letters $P, R$ and $D$ indicate particle, recrystallized and deformed sites, respectively. Single kink (a) and double kink (b) methods for pulling the recrystallization front off the particles are described in the text.

Inspection of Fig. 18(b) shows that this can be achieved by the influence of a third grain B, that may be either deformed or recrystallized. The arrival of a step from the upper grain B, shown by the arrow, when it has reached $D_{9}$, will allow the $D$ site at the edge, $D_{e}$, to switch to $R$ and provide the needed step for $D_{2}$ and then $D_{3}$ to switch and the boundary to escape from the particle. This analysis is identical to that of grain growth and immediately suggests that recrystallization with $H / J<1$, is only that of grain growth biased in favor of those grains that have grown larger by virtue of their lower stored energy. The conclusion that recrystallization is insensitive to $H$ when $H / J<1$ was verified by repeating simulations with $f=5 \%$ and 200 embryos at $H / J=0.2$ and $H / J=0.99$. The results were identical to this for $H / J=0.1$, that is, the recrystallization did not go to completion and the kinetics were identical to those seen in Fig. 12.

For recrystallization with $H / J>1$, since it has been shown that the particles exert a negligible drag on the moving boundaries, it then follows that the growth kinetics should be unaffected by the area fraction of particles. This result was indeed found in these simulations, Fig. 10, and this confirms the established viewpoint on recrystallization that particles appear to have a negligible effect on the growth kinetics, even though they strongly influence nucleation.

This result for recrystallization growth, driven by significant values of stored energy, strongly contrasts with the dramatic effect that particles exert on the coarsening process driven by the grain boundary energy-that is during grain growth [16, 33]. In grain growth, boundaries attach themselves strongly to particles as seen in Fig. 18, and thereby remove the curvature needed for coarsening driven by interfacial energy. This effect was repeatedly observed in the present study-in the inhibited grain growth seen in the deformed grains, Figs 14 and 17, and in the inhibited growth in the new grains seen after recrystallization, Figs 9(c), 10(c), 17 and 18. The origin of this effect is shown by the rise in particle density on the grain boundaries above the random value during grain growth, Fig. 7. This result is explored in more detail in a separate study $[16,33]$.

Perhaps the most striking evidence for the strong influence of particles on grain growth in the present study is the results of the behavior of the unrecrystallized and the new grains in simulations with $H / J<1$. Here the growth of the new grains was pinned out just as was the growth of the deformed grains as the grain boundaries migrated until pinned by the particles, Figs 16 and 18(a). For the lowest area fraction, particle pinning did not occur so that recrystallization went slowly to completion, Fig. 12. That this was mainly a grain growth effect is shown by Figs 16 and 19. Figure 16 shows that the new grains are growing into a residue of smaller deformed grains. Figure 19 shows the relative grain growth rates of new grains and all grains. It is clear that the small stored energy advantage provides sufficient bias so that the mean size of the new grains grows faster than that of the old grains, giving a situation closer to abnormal grain growth than true primary recrystallization. As suspected from the previous analysis the new grains as

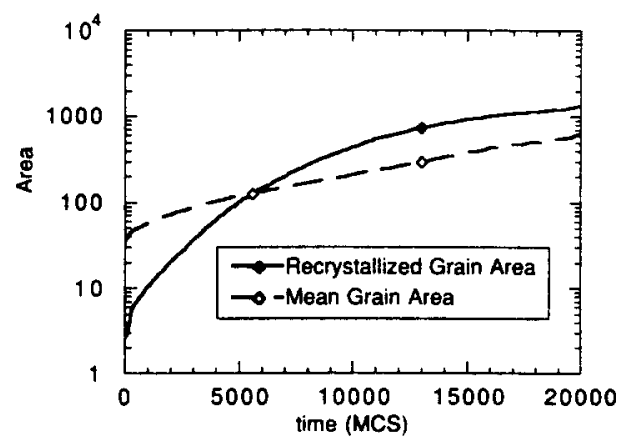

Fig. 19. Mean grain size (open symbols) and mean recrystallized grain size (filled symbols) as a function of time for stored energy of $H / J=0.1$, a particle area fraction of $2.5 \%$ and 200 site saturated nuclei. 
well as the old grains showed a much higher density of particles at their boundaries, about $1 \%$, compared to the random value, $f=0.25 \%$.

A quantitative comparison of recrystallization with $H / J<1$ with pure grain growth is instructive. For a particle fraction of $1.25 \%$, the pinned grain area (for an initial $\langle A\rangle=40$ ) is 235 . For recrystallization with 200 embryos to go to completion with all embryos surviving, the final grain area would be $\langle A\rangle=200$. At $H / J<1$ and a particle fraction of $1 \%$, recrystallization nearly goes to completion, whereas at a particle fraction of $0.25 \%$, recrystallization does go to completion. This would appear to suggest a simple comparison between recrystallization and grain growth where the criterion for the success of recrystallization (at low stored energies) is that the final grain size (based on the initial number of embryos) be smaller than the limiting grain size for the applicable particle fraction. The actual recrystallization behavior is, however, more complex. The final mean area of the recrystallized grains for $H / J=0.1,200 \mathrm{em}$ bryos and a particle fraction of $1 \%$ is $>500$, which is much larger than the corresponding mean area for grain growth alone. This shows that the behavior at low stored energies is not simply that of grain growth but is significantly altered by the bias induced by the stored energy field.

Figure 20 shows a comparison of the grain size distributions plotted as the relative frequency vs the logarithm of the radius relative to the mean radius. Two plots are given: one for grain growth without particles, which shows a Rayleigh distribution [10], and two for recrystallized microstructures containing 2000 particles (5\% area fraction), with $H / J=1$ and two different numbers of embryos at zero time. Whereas the plot for the large number of embryos (1000 embryos) is similar to that of grain growth, the data for a small number of embryos (100 embryos) shows a distribution that is much more sharply

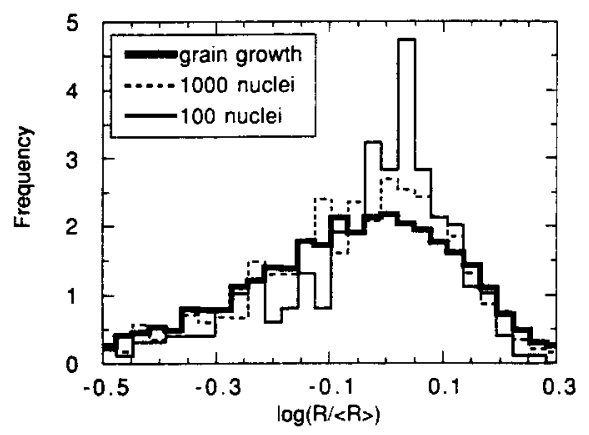

Fig. 20. Grain size distribution plotted as the relative frequency vs normalized grain size for grain growth in the absence of particles and for recrystallization with $H / J=1.01$, with a particle area fraction of $5 \%$ and two different nucleation densities. For a high nucleation density (1000 nuclei) the distribution is close to that of normal grain growth. For a low nucleation density (100 nuclei), however, the distribution is narrower, more peaked and has a smaller upper cut-off size in size. peaked at $\langle R\rangle$ and has a smaller upper cut-off radius. When the nucleation density is high, recrystallization goes to completion and yields a grain size that is smaller than the normal grain growth particle pinned grain size and hence grain growth follows, leading to a grain size distribution that is close to that expected for normal grain growth. However, when the nucleation density is low, the recrystallized grain size is larger than the normal grain growth particle pinned grain size and hence no grain growth follows the completion of recrystallization. Therefore Fig. 20 demonstrates that recrystallization leads to a narrower grain size distribution than does normal grain growth. This is an agreement with the observations made on grain shapes above, Fig. 17.

\section{CONCLUSIONS}

- During recrystallization simulation with sufficient stored energy $(H / J>1)$ the recrystallization front can readily bypass particles. This leads to a near random density of particles on grain boundaries, as has been observed experimentally. Under these circumstances the recrystallizai on growth kinetics are unaffected by particles. The overall kinetics are affected, however, by heterogeneous nucleation on particles.

- At intermediate stored energies, $1<H / J<2$, recrystallization depends on the existence of a prior grain structure. This is because there is insufficient stored energy to nucleate kinks at a recrystallization front and growth of a recrystallizing grain occurs along prior boundaries [7].

- At low stored energies $(H / J<1)$ the recrystallization front is strongly pinned by particles which leads to a much higher than random density of particles on the recrystallization front. In these circumstances recrystallization growth is strongly inhibited which usually results in incomplete recrystallization. However, for low stored energies $(H / J<1)$ and when small particle fractions are present, recrystallization may go to completion because pinning does not occur until after the transformation is complete.

- The presence of small stored energies $(H / J<1)$ gives the recrystallized grains a growth advantage, such that the recrystallized grains can grow (at low particle fractions) much larger than the deformed matrix grains. This is sufficient to drive the recrystallizing grains past particles, but only if the matrix grain size is much smaller than the interparticle spacing. In other words, recrystallization at low stored energy and at very low particle fractions is similar to abnormal grain growth.

- As shown previously, grain boundaries undergoing curvature driven growth (both in the deformed state and after complete recrystallization) rapidly acquire a higher than random density of particles which then inhibits grain growth. 
- If the recrystallized grain size is smaller than the normal grain growth particle pinned grain size, grain growth continues until pinning occurs and the microstructure is a normal grain growth micro- structure. However, when the recrystallized grain size is large compared to the normal grain growth particle pinned grain size, particle pinning occurs almost immediately following the completion of recrystallization thus preserving the non-compact grain shapes and sharply peaked grain size distribution that are characteristic of randomly distributed nuclei.

- When the stored energy is low $(H / J<2)$, increasing the number of particles also increases the number of nuclei that are super-critical and survive because the particles act as heterogeneous nucleation sites.

- Analysis of recrystallization in particle containing materials suggests that there are two limiting values of particle drag; a low (Zener) value with a random density of particles and a much higher value if particles have become highly correlated with the recrystallization front (as in grain growth). The simulation results show both these behaviors, depending on the $H / J$ ratio. Experimental studies appear to show only the lower particle drag (Zener) as studied by Ashby et al. [21].

Acknowledgements - The authors would like to acknowledge the contribution of G. S. Grest to the simulation technique. This work was supported in part by the U.S. Department of Energy.

\section{REFERENCES}

1. R. D. Doherty and J. W. Martin, J. Inst. Metals 91,332 (1962).

2. N. Hansen, Mem. Sci. Rev. Métall. 72, 189 (1975).

3. F. J. Humphreys, Metals Sci. 13, 136 (1979).

4. U. Koster and E. Hornbogen, in Recrystallization of Metallic Materials (edited by F. Hassner), p. 159. Dr. Riederer, Stuttgart (1978).

5. F. J. Humphreys, Acta metall. 25, 1323 (1977).

6. D. J. Srolovitz, G. S. Grest and M. P. Anderson, Acta metall. 34, 1833 (1986).

7. D. J. Srolovits, G. S. Grest, M. P. Anderson and A. D. Rollett, Acta metall. 36, 2115 (1988).

8. A. D. Rollet, D. J. Srolovitz, R. D. Doherty and M. P. Anderson, Acta metall. 27, 627 (1989).

9. M. P. Anderson, D. J. Srolovitz, P. S. Sahni and G. S. Grest, Acta metall. 32, 783 (1984).

10. D. J. Srolovitz, M. P. Anderson. P. S. Sahni and G. S. Grest, Acta metall. 32, 793 (1984).
11. D. J. Srolovitz, M. P. Anderson, G. S. Grest and P. S. Sahni, Acta metall. 32, 1429 (1984).

12. G. S. Grest, D. J. Srolovit and M. P. Anderson, Acta metall. 33, 509 (1985).

13. D. J. Srolovitz, G. S. Grest and M. P. Anderson, Acta metall. 33, 2233 (1985).

14. A. D. Rollett, D. J. Srolovitz and M. P. Anderson, Acta metall. 37, 1227 (1989).

15. M. P. Anderson, G. S. Grest and D. J. Srolovitz, Phil. Mag. B 59, 293 (1989).

16. R. D. Doherty, K. Li, K. Kashyup, A. D. Rollett and M. P. Anderson, in Materials Architecture (edited by J. B. Bilde-Sorensen et al.), 10th Risø Symp., p. 31. Risø National Laboratory, Roskilde, Denmark (1989).

17. M. P. Anderson, G. S. Grest, K. Li, R. D. Doherty and D. J. Srolovitz, Scripta metall. 23, 753 (1989).

18. C. Zener, private communication to C. S. Smith, cited in Trans. Am. Inst. Min. Engrs 175, 15 (1948)

19. R. D. Doherty, D. J. Srolovitz. A. D. Rollett and M. P. Anderson, Scripta metall. 21, 675 (1987)

20. M. F. Ashby, in Recrystallization and Grain Growth of Multi-Phase and Particle Containing Materials (edited by N. Hansen et al.), 1st Risø Symp., p. 325. Risø National Laboratory, Roskilde, Denmark (1980).

21. M. F. Ashby, J. Harper and J. Lewis, Trans. Am. Inst. Min. Engrs 245, 413 (1969).

22. D. L. Olgaard and B. Evans, J. Am. Ceram. Soc. 69, C272 (1986).

23. M. Hillert, Acta metall. 36, 3177 (1989).

24. E. E. Underwood, in Quantitative Stereology. p. 85. Addison-Wesley, Reading (1970).

25. R. D. Doherty, in Recrystallization of Metallic Materials (edited by F. Hassner), p. 27. Dr. Riederer, Stuttgart (1978).

26. D. Nobili and R. DeMaria, J. nucl. Mater. 17, 5 (1965).

27. P. R. Howell and J. V. Bee, in Recrystallization and Grain Growth of Multi-Phase and Particle Containing Materials (edited by N. Hansen et al.), Ist Risø Symp., p. 171. Risø National Laboratory, Roskilde, Denmark (1980).

28. P. L. Morris and M. D. Ball, in Recrustallization and Grain Growth of Multi-Phase and Particle Containing Materials (edited by N. Hansen et al.), 1st Risø Symp. p. 97. Risø National Laboratory, Roskilde. Denmark (1980).

29. U. Lotter, W. Müschenborn and E. Thiemann, in Recrystallization and Grain Growth of Multi-Phase and Particle Containing Materials (edited by N. Hansen et al.), 1st Risø Symposium, p. 133. Risø National Laboratory, Roskilde, Denmark (1980).

30. R. D. Doherty, Metals Sci. 16, 1 (1982).

31. B. Hutchinson and B. J. Duggan, Metals Sci. 12, 372 (1978).

32. I. Baker and J. W. Martin, J. Mater. Sci. 15, 1533 (1980).

33. M. F. Ashby and R. M. A. Centamore, Acta metall. 16, 1081 (1968).

34. R. D. Doherty, A. D. Rollett, D. J. Srolovitz and M. P. Anderson, to be published. 